\section{Banking, insurance}

\section{Other banks balance sheet}

(Table 23.3)

The table includes the business of all monthly and quarterly reporting banks in the UK.

The Channel Islands and Isle of Man are not treated as part of the UK for statistical purposes. Banking institutions in the Channel Islands and Isle of Man no longer have the option of being within the UK banking sector and their business, along with the business of offshore island branches of UK mainland banks, is excluded from the figures within this table. Additionally, the business of the UK banking sector with offshore island residents and entities are classified as 'nonresidents'.

The table also contains details of business with building societies.

The aggregate balance sheet of the banking sector is reported on an accrual basis (accrued amounts that are payable and receivable are shown under liabilities and assets respectively). Additionally, acceptances are shown under both liabilities and assets.

The balance sheet of the Banking Department of the Bank of England is excluded from this table, and other banks business with the Issue Department is classified as 'UK banks'.

Data for 1999 reflect the acquisition of Birmingham Midshires Building Society by Halifax during that year.

Data for the end of 2000 reflect the entry of Bradford and Bingley plc to the banking sector during the year. Data for the end of 2000 also reflect the new reporting during the year of agency business as a result of collateral management via repurchase agreements (repos) and reverse repos.

\section{Bank lending to, and bank deposits from, UK residents}

(Tables 23.4 and 23.5)

These are series statistics based on the Standard Industrial Classification (SIC) 1992 (which was revised slightly in 2003).

Table 23.4. Until the third quarter of 2007, the analysis of lending covered loans, advances (including under reverse repos), finance leasing, acceptances and facilities (all in Sterling and other currencies) provided by reporting banks to their UK resident non-bank non-building society customers, as well as bank holdings of sterling and euro commercial paper issued by these resident customers. Following a review of statistical data collected, acceptances and holdings of sterling and euro commercial paper are no longer collected at the industry level detail with effect from fourth quarter 2007 data. Total lending therefore reflects loans and advances (including under reverse repos) only, from fourth quarter 2007 data.

Table 23.5 includes borrowing under sale and repo.

Adjustments for transit items are not included.

Figures for both tables are supplied by monthly reporting banks and grossed to cover quarterly reporters. Following the transition of building societies' statistical reporting from the Financial Services Authority to the Bank of England on 1st January 2008, both tables will include data reported by building societies from the first quarter of 2008 onwards. They exclude lending to building societies and to residents of the Channel Islands and Isle of Man.

\section{Building societies}

(Table 23.13)

Building society figures are sourced from societies' annual returns and for each year relate to accounting years ending on dates between 1 February and 31 January of the following year. Figures are society-only as opposed to group consolidated.

\section{Consumer credit}

(Table 23.14)

Figures for net lending refer to changes in amounts outstanding adjusted to remove distortions caused by

revaluations of debt outstanding, such as write-offs. Class 3 loans are advanced under the terms of the Building Societies Act 1986. 
A high proportion of credit advanced in certain types of agreement, notably on credit cards, is repaid within a month. This reflects use of such agreements as a method of payment rather than a way of obtaining credit. As from December 2006 the Bank of England has ceased to update the separate data on consumer credit provided by other specialist lenders, retailers and insurance companies previously contained in these tables. These categories have been merged into 'other consumer credit lenders'. 


\section{Banking, insurance}

\section{Bank of England Balance Sheet}

Liabilities and assets outstanding at end of period

$£$ million

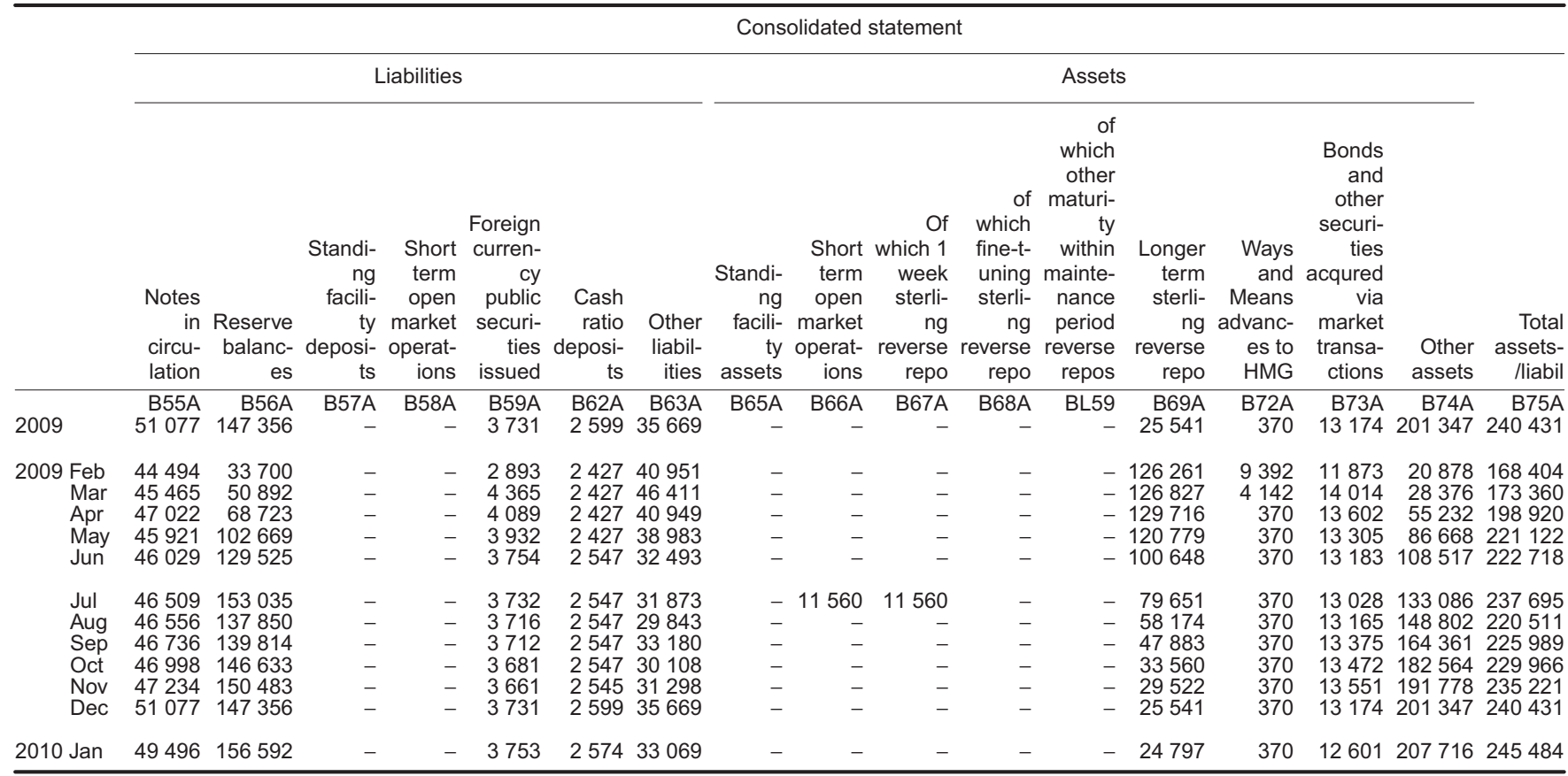

Issue Department

Liabilities

Assets

\begin{tabular}{|c|c|c|c|c|c|c|c|c|c|c|}
\hline & & & & \multirow[b]{2}{*}{$\begin{array}{r}\text { Total } \\
\text { assets/liabi- } \\
\text { lities }\end{array}$} \\
\hline & $\begin{array}{r}\text { Notes in } \\
\text { circu- lation }\end{array}$ & $\begin{array}{r}\text { Notes in } \\
\text { Banking } \\
\text { Departemnt }\end{array}$ & $\begin{array}{r}\text { Short term } \\
\text { open market } \\
\text { operations }\end{array}$ & $\begin{array}{r}\text { Of which } 1 \\
\text { week sterling } \\
\text { reverse repo }\end{array}$ & $\begin{array}{r}\text { of which } \\
\text { fine-tuning } \\
\text { sterling } \\
\text { reverse repo }\end{array}$ & $\begin{array}{r}\text { Longer term } \\
\text { sterling } \\
\text { reverse repo }\end{array}$ & $\begin{array}{r}\text { Ways and } \\
\text { Means } \\
\text { advances to } \\
\text { HMG }\end{array}$ & $\begin{array}{r}\text { Bonds and } \\
\text { other } \\
\text { securities } \\
\text { acqured via } \\
\text { market } \\
\text { transactions }\end{array}$ & Other assets & \\
\hline 2009 & $\begin{array}{r}\text { AEFA } \\
51077\end{array}$ & $\begin{array}{r}\mathrm{AEFB} \\
-\end{array}$ & $\begin{array}{r}\mathrm{BL} 29 \\
-\end{array}$ & $\begin{array}{r}\mathrm{BL} 32 \\
-\end{array}$ & $\begin{array}{r}\mathrm{BL} 33 \\
-\end{array}$ & $\begin{array}{r}\text { BL34 } \\
20395\end{array}$ & $\begin{array}{r}\text { B54A } \\
370\end{array}$ & $\begin{array}{l}\text { BL35 } \\
5439\end{array}$ & $\begin{array}{r}\text { BL36 } \\
24874\end{array}$ & $\begin{array}{r}\text { BL37 } \\
51077\end{array}$ \\
\hline $\begin{array}{r}2009 \text { Mar } \\
\text { Apr } \\
\text { May } \\
\text { Jun }\end{array}$ & $\begin{array}{l}45465 \\
47022 \\
45921 \\
46029\end{array}$ & $\begin{array}{l}- \\
- \\
- \\
-\end{array}$ & $\begin{array}{l}- \\
- \\
-\end{array}$ & $\begin{array}{l}- \\
- \\
- \\
-\end{array}$ & $\begin{array}{l}- \\
- \\
- \\
-\end{array}$ & $\begin{array}{l}9799 \\
9719 \\
9719 \\
9769\end{array}$ & $\begin{array}{r}4142 \\
370 \\
370 \\
370\end{array}$ & $\begin{array}{l}5435 \\
5433 \\
5433 \\
5417\end{array}$ & $\begin{array}{l}26088 \\
31501 \\
30400 \\
30474\end{array}$ & $\begin{array}{ll}45 & 465 \\
47 & 022 \\
45 & 921 \\
46 & 029\end{array}$ \\
\hline $\begin{array}{l}\text { Jul } \\
\text { Aug } \\
\text { Sep } \\
\text { Oct } \\
\text { Nov } \\
\text { Dec }\end{array}$ & $\begin{array}{l}46509 \\
46556 \\
46736 \\
46998 \\
47234 \\
51077\end{array}$ & $\begin{array}{l}- \\
- \\
- \\
- \\
-\end{array}$ & $\begin{array}{r}11560 \\
- \\
- \\
- \\
- \\
-\end{array}$ & $\begin{array}{r}11560 \\
- \\
- \\
- \\
- \\
-\end{array}$ & $\begin{array}{l}- \\
- \\
- \\
- \\
-\end{array}$ & $\begin{array}{r}9770 \\
13405 \\
18517 \\
23740 \\
22799 \\
20395\end{array}$ & $\begin{array}{l}370 \\
370 \\
370 \\
370 \\
370 \\
370\end{array}$ & $\begin{array}{ll}5 & 417 \\
5 & 417 \\
5 & 493 \\
5 & 493 \\
5 & 493 \\
5 & 439\end{array}$ & $\begin{array}{l}19392 \\
27364 \\
22356 \\
17394 \\
18571 \\
24874\end{array}$ & $\begin{array}{l}46509 \\
46556 \\
46736 \\
46998 \\
47234 \\
51077\end{array}$ \\
\hline $\begin{array}{r}2010 \text { Jan } \\
\text { Feb }\end{array}$ & $\begin{array}{l}49496 \\
49486\end{array}$ & $\begin{array}{l}- \\
-\end{array}$ & $\begin{array}{l}- \\
. .\end{array}$ & $\begin{array}{l}- \\
. .\end{array}$ & $\begin{array}{l}- \\
. .\end{array}$ & $\begin{array}{r}19712 \\
. .\end{array}$ & $\begin{array}{r}370 \\
. .\end{array}$ & $\begin{array}{r}5439 \\
. .\end{array}$ & $\begin{array}{r}23975 \\
. .\end{array}$ & 49496 \\
\hline
\end{tabular}

\begin{tabular}{|c|c|c|c|c|c|c|c|c|c|c|c|c|c|c|c|}
\hline Feb & & 486 & - & & .. & .. & & .. &.. & & .. & .. & & .. & .. \\
\hline & \multicolumn{15}{|c|}{ Banking Department } \\
\hline & \multicolumn{6}{|c|}{ Liabilities } & \multicolumn{8}{|c|}{ Assets } & \multirow[b]{2}{*}{$\begin{array}{r}\text { Total } \\
\text { assets/l- } \\
\text { iabilitie }\end{array}$} \\
\hline & $\begin{array}{r}\text { Reserve } \\
\text { balances }\end{array}$ & $\begin{array}{r}\text { Standing } \\
\text { facility } \\
\text { deposits }\end{array}$ & $\begin{array}{r}\text { Short } \\
\text { term open } \\
\text { market } \\
\text { operatio- } \\
\text { ns }\end{array}$ & $\begin{array}{r}\text { Foreign } \\
\text { currency } \\
\text { public } \\
\text { securiti- } \\
\text { es issued }\end{array}$ & $\begin{array}{r}\text { Cash } \\
\text { ratio } \\
\text { deposits }\end{array}$ & $\begin{array}{r}\text { Other } \\
\text { liabilit- } \\
\text { ies }\end{array}$ & $\begin{array}{r}\text { Standing } \\
\text { facility } \\
\text { assets }\end{array}$ & $\begin{array}{r}\text { Short } \\
\text { term open } \\
\text { market } \\
\text { operatio- } \\
\text { ns }\end{array}$ & $\begin{array}{r}\text { Of which } \\
1 \text { week } \\
\text { sterling } \\
\text { reverse } \\
\text { repo }\end{array}$ & $\begin{array}{r}\text { of which } \\
\text { fine-tun- } \\
\text { ing } \\
\text { sterling } \\
\text { reverse } \\
\text { repo }\end{array}$ & $\begin{array}{r}\text { Longer } \\
\text { term } \\
\text { sterling } \\
\text { reverse } \\
\text { repo }\end{array}$ & $\begin{array}{r}\text { Bonds and } \\
\text { other } \\
\text { securiti- } \\
\text { es } \\
\text { acqured } \\
\text { via } \\
\text { market } \\
\text { transact- } \\
\text { ions }\end{array}$ & $\begin{array}{r}\text { Bank of } \\
\text { England } \\
\text { notes }\end{array}$ & $\begin{array}{r}\text { Other } \\
\text { assets }\end{array}$ & \\
\hline 2009 & $\begin{array}{r}\text { BL38 } \\
147356\end{array}$ & $\begin{array}{r}\text { BL39 } \\
-\end{array}$ & $\begin{array}{r}\text { BL42 } \\
-\end{array}$ & $\begin{array}{r}\text { BL43 } \\
3731\end{array}$ & $\begin{array}{r}\text { BL44 } \\
2599\end{array}$ & $\begin{array}{r}\text { BL45 } \\
60542\end{array}$ & $\begin{array}{r}\text { BL47 } \\
-\end{array}$ & $\begin{array}{r}\text { BL48 } \\
-\end{array}$ & $\begin{array}{r}\text { BL49 } \\
-\end{array}$ & $\begin{array}{r}\text { BL52 } \\
-\end{array}$ & $\begin{array}{r}\text { B3J2 } \\
5146\end{array}$ & $\begin{array}{l}\text { BL53 } \\
7735\end{array}$ & $\begin{array}{r}\text { BL54 } \\
-\end{array}$ & $\begin{array}{r}\text { BL55 } \\
201347\end{array}$ & $\begin{array}{r}\text { BL56 } \\
214228\end{array}$ \\
\hline $\begin{array}{c}2009 \text { Feb } \\
\text { Mar } \\
\text { Apr } \\
\text { May } \\
\text { Jun }\end{array}$ & $\begin{array}{r}33700 \\
50892 \\
68723 \\
102669 \\
129525\end{array}$ & $\begin{array}{l}- \\
- \\
- \\
- \\
-\end{array}$ & $\begin{array}{l}- \\
- \\
- \\
- \\
-\end{array}$ & $\begin{array}{l}2893 \\
4365 \\
4089 \\
3932 \\
3754\end{array}$ & $\begin{array}{l}2427 \\
2427 \\
2427 \\
2427 \\
2547\end{array}$ & $\begin{array}{l}61311 \\
72499 \\
72450 \\
69383 \\
62966\end{array}$ & $\begin{array}{l}- \\
- \\
- \\
- \\
-\end{array}$ & $\begin{array}{l}- \\
- \\
- \\
- \\
-\end{array}$ & $\begin{array}{l}- \\
- \\
- \\
-\end{array}$ & $\begin{array}{l}- \\
- \\
- \\
- \\
-\end{array}$ & $\begin{array}{r}116463 \\
-117028 \\
-119997 \\
-111060 \\
-\quad 90879\end{array}$ & $\begin{array}{l}6929 \\
8579 \\
8169 \\
7872 \\
7766\end{array}$ & $\begin{array}{l}- \\
- \\
- \\
-\end{array}$ & $\begin{array}{r}20878 \\
28376 \\
55232 \\
86668 \\
108517\end{array}$ & $\begin{array}{l}144270 \\
153983 \\
183399 \\
205600 \\
207162\end{array}$ \\
\hline $\begin{array}{l}\text { Jul } \\
\text { Aug } \\
\text { Sep } \\
\text { Oct } \\
\text { Nov } \\
\text { Dec }\end{array}$ & $\begin{array}{l}153035 \\
137850 \\
139814 \\
146633 \\
150483 \\
147356\end{array}$ & $\begin{array}{l}- \\
- \\
- \\
- \\
- \\
-\end{array}$ & $\begin{array}{l}- \\
- \\
- \\
- \\
-\end{array}$ & $\begin{array}{ll}3 & 732 \\
3 & 716 \\
3 & 712 \\
3 & 681 \\
3 & 661 \\
3 & 731\end{array}$ & $\begin{array}{ll}2 & 547 \\
2 & 547 \\
2 & 547 \\
2 & 547 \\
2 & 545 \\
2 & 599\end{array}$ & $\begin{array}{l}51265 \\
57207 \\
55536 \\
47503 \\
49869 \\
60542\end{array}$ & $\begin{array}{l}- \\
- \\
- \\
- \\
- \\
-\end{array}$ & $\begin{array}{l}- \\
- \\
- \\
- \\
- \\
-\end{array}$ & $\begin{array}{l}- \\
- \\
- \\
- \\
- \\
-\end{array}$ & $\begin{array}{l}- \\
- \\
- \\
- \\
- \\
-\end{array}$ & $\begin{array}{r}69881 \\
-\quad 44769 \\
-\quad 29366 \\
-\quad 9820 \\
6723 \\
5146\end{array}$ & $\begin{array}{ll}7 & 611 \\
7 & 748 \\
7 & 882 \\
7 & 979 \\
8 & 058 \\
7 & 735\end{array}$ & $\begin{array}{l}- \\
- \\
- \\
- \\
-\end{array}$ & $\begin{array}{r}133086 \\
148802 \\
164361 \\
182564 \\
191778 \\
201347\end{array}$ & $\begin{array}{l}210578 \\
201319 \\
201609 \\
200363 \\
206559 \\
214228\end{array}$ \\
\hline 2010 Jan & 156592 & - & - & 3753 & 2574 & 57044 & - & - & - & - & 5085 & 7163 & & 207716 & 219964 \\
\hline
\end{tabular}


Banking, insurance

232 Value of inter-bank clearings

- United Kingdom

2004

Bulk paper clearings

Cheque and Credit Clearing Company Cheques $^{1}$

Euro Debits

Credits

Inter-bank Cheque and Credit

उT 80

KCYZ

JT8P

$\begin{array}{rr}1111 & 1062 \\ . . & . . \\ 63 & 57 \\ 1174 & 1119\end{array}$

1076

56

119

1132

58
1215

1076

3
52

1130

$£$ billion

High-value clearings

Faster Payments Scheme ${ }^{3}$

CHAPS Sterling

JT 8N

KCZB

52348

$5267 \ddot{2}$

59437

69352

33
73626

64617

Electronic clearing (BACS)

Standing Orders/Direct Credits ${ }^{2}$

Direct Debits

JT8J

JT8L

JT8M

Euro Direct Credits

KCZC

Total Bacs

JT8Q
2133

750

2883

56405
2353

797

3150

56941
2584

2812
845

3429

63998

74263

3006

935

5
3946

88127

Source: APACS - The UK payments association: 02077116223

1 Figures for 2004 - 2007 include Euro Debits.

2 Figures for 2004 - 2007 include Euro Direct Credits.

3 The UK Faster Payments Services was launched on 27th May 2008. 


\section{Monetary Financial Institutions \\ 2.3. (Excluding Central Bank) Balance sheet, Liabilities and Assets \\ Amount outstanding at end of period}

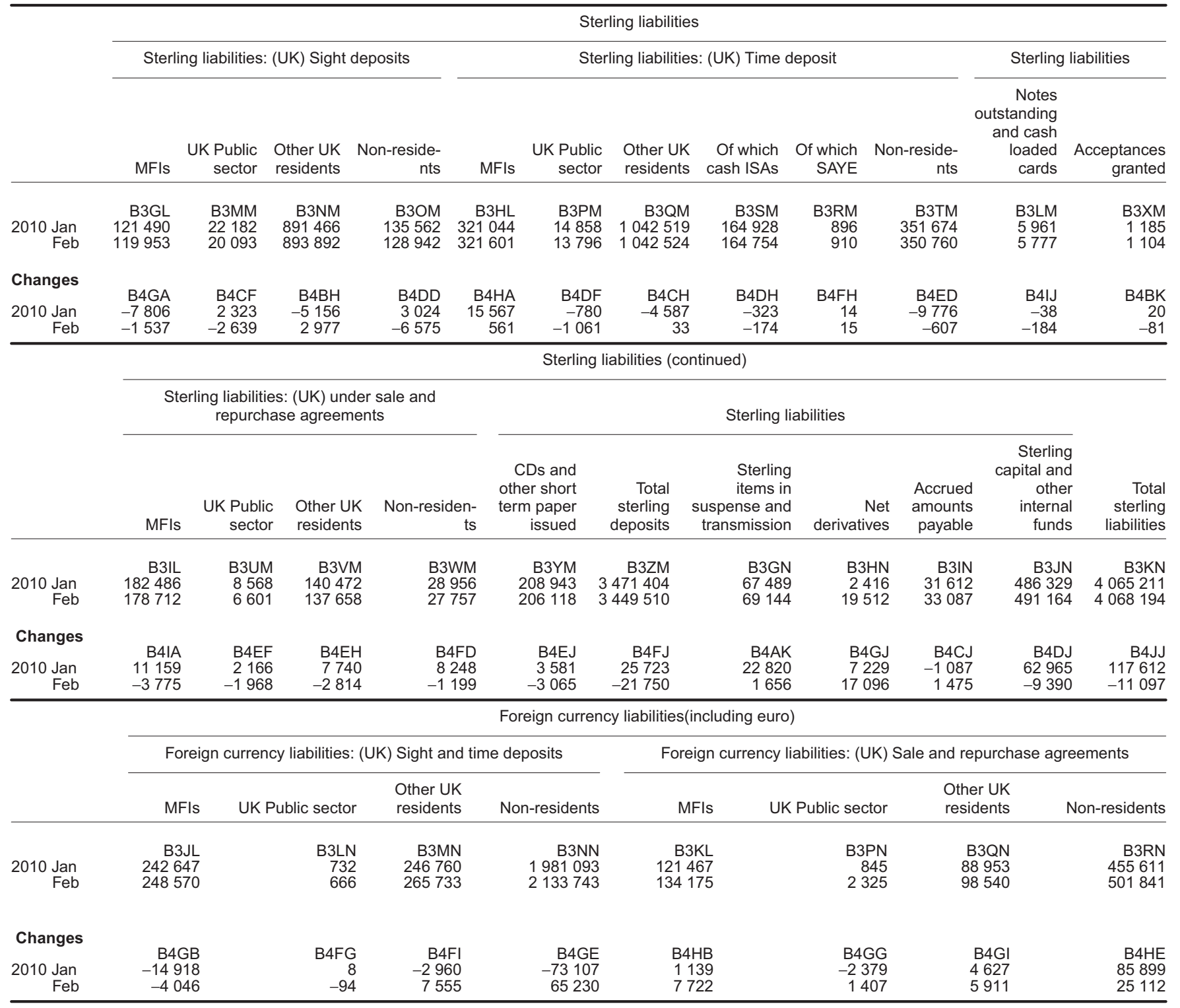


Foreign currency liabilities (including euro)(continued)

\begin{tabular}{|c|c|c|c|c|c|c|c|c|c|}
\hline & $\begin{array}{r}\text { Acceptances } \\
\text { granted }\end{array}$ & $\begin{array}{r}\text { CDs and other } \\
\text { short term } \\
\text { paper issued }\end{array}$ & $\begin{array}{r}\text { Total foreign } \\
\text { currency } \\
\text { deposits }\end{array}$ & $\begin{array}{l}\text { Items in } \\
\text { suspense and } \\
\text { transmission }\end{array}$ & Net derivatives & $\begin{array}{r}\text { Accrued amounts } \\
\text { payable }\end{array}$ & $\begin{array}{r}\text { Capital and } \\
\text { other internal } \\
\text { funds }\end{array}$ & $\begin{array}{r}\text { Total foreign } \\
\text { currency } \\
\text { liabilities }\end{array}$ & $\begin{array}{r}\text { Total } \\
\text { liabilities }\end{array}$ \\
\hline $\begin{array}{l}2010 \text { Jan } \\
\text { Feb }\end{array}$ & $\begin{array}{l}\text { B3KQ } \\
1049 \\
1200\end{array}$ & $\begin{array}{r}\text { B3SN } \\
770717 \\
798510\end{array}$ & $\begin{array}{r}\text { B3TN } \\
3909874 \\
4185303\end{array}$ & $\begin{array}{r}\text { B3UN } \\
225284 \\
232634\end{array}$ & $\begin{array}{r}\text { B3VN } \\
-52004 \\
-76526\end{array}$ & $\begin{array}{l}\text { B3WN } \\
26523 \\
26568\end{array}$ & $\begin{array}{r}\text { B3XN } \\
135873 \\
136379\end{array}$ & $\begin{array}{r}\text { B3YN } \\
4245550 \\
4504357\end{array}$ & $\begin{array}{r}\text { B3ZN } \\
8310762 \\
8572551\end{array}$ \\
\hline
\end{tabular}

\begin{tabular}{|c|c|c|c|c|c|c|c|c|c|}
\hline Changes & & & & & & & & & \\
\hline $\begin{array}{l}2010 \text { Jan } \\
\text { Feb }\end{array}$ & $\begin{array}{r}\text { B4HM } \\
-26 \\
101\end{array}$ & $\begin{array}{r}\text { B4CM } \\
14096 \\
-10388\end{array}$ & $\begin{array}{r}\text { B4DM } \\
12379 \\
98510\end{array}$ & $\begin{array}{r}\text { B4GM } \\
124670 \\
3036\end{array}$ & $\begin{array}{r}\text { B4EM } \\
3423 \\
-26317\end{array}$ & $\begin{array}{r}\text { B4AM } \\
-3623 \\
-386\end{array}$ & $\begin{array}{l}\text { B4BM } \\
2954 \\
5121\end{array}$ & $\begin{array}{r}\text { B4FM } \\
139804 \\
79964\end{array}$ & $\begin{array}{r}\text { B4JM } \\
257415 \\
68866\end{array}$ \\
\hline
\end{tabular}

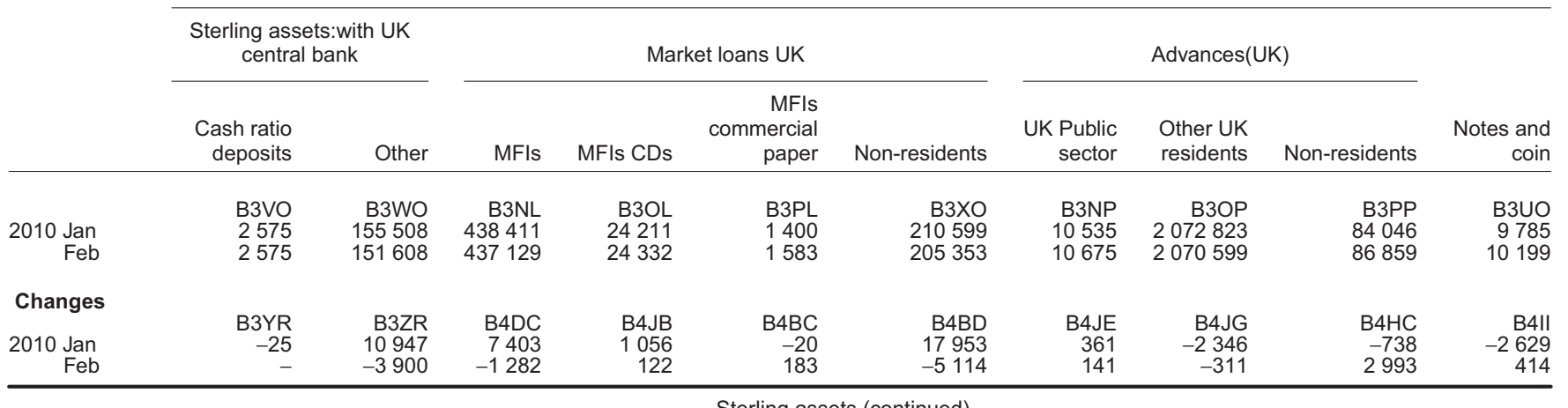

\begin{tabular}{|c|c|c|c|c|c|c|c|c|c|c|c|c|}
\hline & \multicolumn{12}{|c|}{ Sterling assets (continued) } \\
\hline & \multicolumn{4}{|c|}{ Sterling assets: (UK) Acceptances granted } & \multicolumn{4}{|c|}{ Bills (UK) } & \multicolumn{4}{|c|}{$\begin{array}{l}\text { Claims under sale and repurchase agreements } \\
\text { (UK) }\end{array}$} \\
\hline & MFIs & $\begin{array}{r}\text { UK Public } \\
\text { sector }\end{array}$ & $\begin{array}{l}\text { Other UK } \\
\text { residents }\end{array}$ & $\begin{array}{r}\text { Non-reside- } \\
\text { nts }\end{array}$ & $\begin{array}{r}\text { Treasury } \\
\text { bills }\end{array}$ & MFIs bills & $\begin{array}{l}\text { Other UK } \\
\text { residents }\end{array}$ & $\begin{array}{r}\text { Non-reside- } \\
\text { nts }\end{array}$ & MFIs & $\begin{array}{r}\text { UK Public } \\
\text { sector }\end{array}$ & $\begin{array}{l}\text { Other UK } \\
\text { residents }\end{array}$ & $\begin{array}{r}\text { Non-reside- } \\
\text { nts }\end{array}$ \\
\hline $\begin{array}{r}2010 \text { Jan } \\
\text { Feb }\end{array}$ & $\begin{array}{r}\text { B3QL } \\
1 \\
1\end{array}$ & $\begin{array}{r}\text { B3YO } \\
- \\
-\end{array}$ & $\begin{array}{r}\text { B3ZO } \\
902 \\
838\end{array}$ & $\begin{array}{r}\text { B3GP } \\
282 \\
265\end{array}$ & $\begin{array}{r}\text { B3HP } \\
22967 \\
16066\end{array}$ & $\begin{array}{r}\text { B3RL } \\
40 \\
40\end{array}$ & $\begin{array}{r}\text { B3IP } \\
186 \\
189\end{array}$ & $\begin{array}{l}\text { B3JP } \\
1604 \\
1444\end{array}$ & $\begin{array}{r}\text { B3SL } \\
167638 \\
163782\end{array}$ & $\begin{array}{r}\text { B3KP } \\
512 \\
20\end{array}$ & $\begin{array}{r}\text { B3LP } \\
124066 \\
117996\end{array}$ & $\begin{array}{r}\text { B3MP } \\
32617 \\
33809\end{array}$ \\
\hline $\begin{array}{l}\text { Changes } \\
2010 \text { Jan } \\
\text { Feb }\end{array}$ & $\begin{array}{r}\text { B4EC } \\
- \\
-\end{array}$ & $\begin{array}{r}\text { B4FF } \\
- \\
-\end{array}$ & $\begin{array}{r}\text { B3TR } \\
46 \\
-64\end{array}$ & $\begin{array}{r}\text { B4GD } \\
-25 \\
-17\end{array}$ & $\begin{array}{r}\text { B4BA } \\
148 \\
-6901\end{array}$ & $\begin{array}{r}\text { B4IB } \\
-39 \\
-\end{array}$ & $\begin{array}{r}\mathrm{B} 4 \mathrm{HG} \\
7 \\
3\end{array}$ & $\begin{array}{r}\text { B4IC } \\
. . \\
. .\end{array}$ & $\begin{array}{r}\text { B4FA } \\
10650 \\
-3856\end{array}$ & $\begin{array}{r}\text { B4BF } \\
-1556 \\
-492\end{array}$ & $\begin{array}{r}\text { B4AH } \\
-1691 \\
-6071\end{array}$ & $\begin{array}{l}\text { B4CD } \\
5949 \\
1192\end{array}$ \\
\hline
\end{tabular}




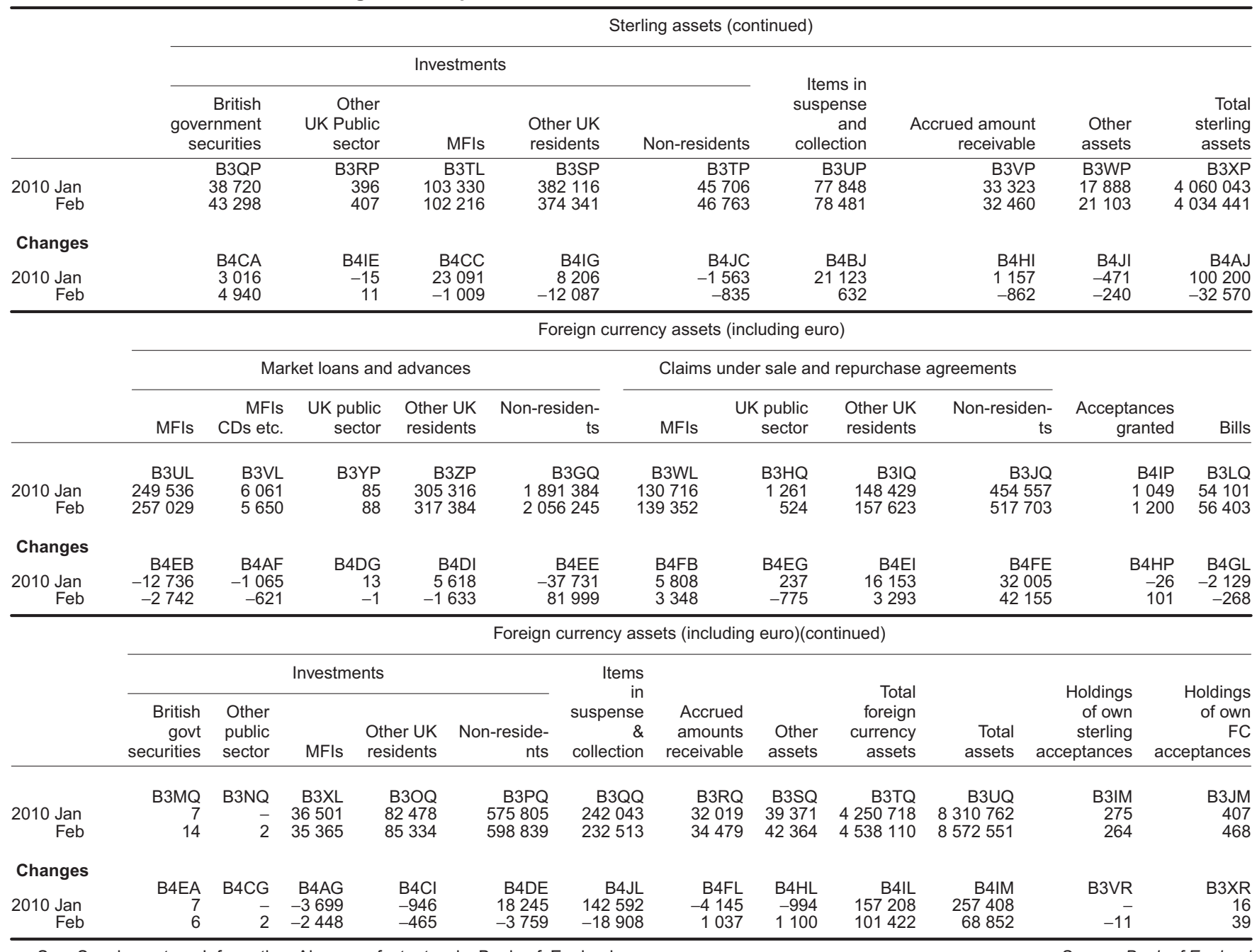

See Supplementary Information Also see footnotes in Bank of England 


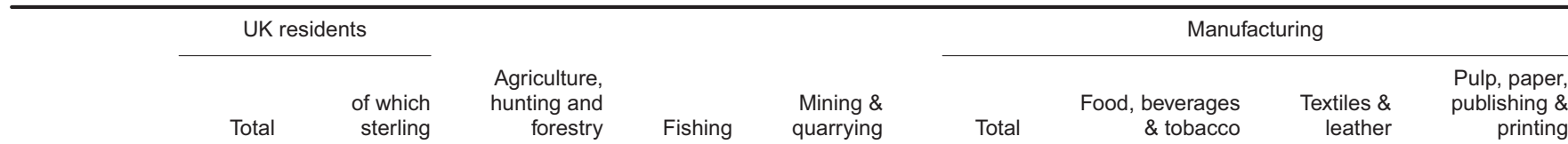

Amounts outstanding (sterling \& other currencies)

Loans \& advances (including under repo \& sterling commercial paper)

\begin{tabular}{rrrrr}
\multicolumn{1}{c}{ TBOA } & TBOB & TBOC & TBOD \\
2006 & 1793840 & 1460380 & 9620 & 413
\end{tabular}

2007

1793840

1460380

413

quarrying

Total \& tobacco printing

Acceptances

\begin{tabular}{lr}
2006 & TBQA \\
2007 & 1190 \\
Total & \\
& TBSA \\
2008 & 263029 \\
2009 & 2514259 \\
\multicolumn{2}{c}{ of which in sterling } \\
\multicolumn{2}{c}{ TBUA } \\
2008 & 2078569 \\
2009 & 2073612
\end{tabular}

TBQB
956

$\begin{array}{rr}\text { TBQC } & \text { TBQD } \\ - & -\end{array}$

$\begin{array}{rr}\text { TBQE } & \text { TBQF } \\ 1 & 104\end{array}$

$\begin{array}{rr}\text { TBOE } & \text { TBOF } \\ 4205 & 47476\end{array}$

$\begin{array}{rr}\text { TBOG } & \text { TBOH } \\ 11434 & 1512\end{array}$

TBOI

476

1512

6405

2073612

$\begin{array}{rrrr}\text { TBSC } & \text { TBSD } & \text { TBSE } & \text { TBSF } \\ 10714 & 356 & 17457 & 65033\end{array}$

65033

11149

347

7060

48789

TBQG

TBQH
28

$\mathrm{TBQ}$

$\begin{array}{lll} & \\ \text { TBUC } & \text { TBUD } & \text { TBUE }\end{array}$

$\begin{array}{llll}10139 & 347 & 2205 & 32684\end{array}$

28730

10673

$$
338
$$

1922

28730

Facilities granted

$2008 \quad 3087455$

$\begin{array}{rrrr}\text { TCAC } & \text { TCAD } & \text { TCAE } & \text { TCAF }\end{array}$

$\begin{array}{rrrr}14302 & 405 & 23383 & 94754\end{array}$

of which in sterling

\begin{tabular}{lcr} 
& TCCA & \\
2008 & 2362723 & \\
2334244 & \\
\hline & & Manu \\
& $\begin{array}{r}\text { Chemicals, } \\
\text { man-made fibres, } \\
\text { rubber \& }\end{array}$ & $\begin{array}{r}\text { Non-metallic } \\
\text { mineral products }\end{array}$
\end{tabular}

TCCC TCCD TCCE TCCF

$\begin{array}{llll}13317 & 444 & 3180 & 49345\end{array}$

45401

..

$\begin{array}{rrr}\text { TBSG } & \text { TBSH } & \text { TBS } \\ 19428 & 1588 & 7735\end{array}$

$\begin{array}{lll}11168 & 1469 & 8302\end{array}$

Manufacturing

2918

11168

8302

rubber \& \& metals

Machinery,
equipment \&
transport

Electrical

medical \& equipment equipment

Othe
manufacturing

Amounts outstanding (sterling \& other currencies)

\begin{tabular}{|c|c|c|c|c|c|c|c|c|}
\hline \\
\hline \multicolumn{9}{|c|}{$\begin{array}{l}\text { Loans \& advances (including under repo \& sterling commercial paper) } \\
\text { TBOJ }\end{array}$} \\
\hline 2006 & 5681 & 6122 & 6678 & 3741 & 5903 & 7075 & 4235 & 20671 \\
\hline 2007 &.. &.. &.. &.. &.. & - & .. & . \\
\hline \multicolumn{9}{|c|}{ Acceptances } \\
\hline & TBQJ & TBQK & TBQL & TBQM & TBQN & TBQO & TBQP & TBQQ \\
\hline 2006 & 4 & 10 & 8 & 14 & 33 & - & - & 15 \\
\hline 2007 & .. & .. & .. & .. & .. & - & .. & .. \\
\hline \multicolumn{9}{|l|}{ Total } \\
\hline & TBSJ & TBSK & TBSL & TBSM & TBSN & TBSO & TBSP & TBSQ \\
\hline 2008 & .. & 8035 & 10604 & .. & 6158 & 9766 & 3964 & 31706 \\
\hline 2009 & .. & 6756 & 7697 & .. & 4852 & 8021 & 3518 & 26333 \\
\hline \multicolumn{9}{|c|}{ of which in sterling } \\
\hline & TBUJ & TBUK & TBUL & TBUM & TBUN & TBUO & TBUP & TBUQ \\
\hline 2008 & 3191 & 3703 & 4968 & 2128 & 4027 & 7606 & 3933 & 30629 \\
\hline 2009 & 2662 & 4017 & 4141 & 1926 & 3565 & 5922 & 3485 & 25611 \\
\hline \multicolumn{9}{|c|}{ Facilities granted } \\
\hline 2008 & .. & 12608 & 17976 & 8811 & 11438 & 18313 & 7802 & 43141 \\
\hline 2009 & .. & 11366 & 15422 & 7296 & 7788 & 15805 & 7233 & 39160 \\
\hline \multicolumn{9}{|c|}{ of which in sterling } \\
\hline & TCCJ & TCCK & TCCL & TCСM & TCCN & TCCO & TCCP & $\mathrm{TCCQ}$ \\
\hline 2008 & 4715 & 5998 & 8771 & 3419 & 5709 & 11698 & 6975 & 40340 \\
\hline 2009 & 4792 & 6293 & 7885 & 3025 & 5344 & 9651 & 6413 & 36495 \\
\hline
\end{tabular}




\begin{tabular}{|c|c|c|c|c|c|c|c|c|}
\hline \multicolumn{4}{|c|}{ Wholesale and retail trade } & & & \multicolumn{3}{|c|}{$\begin{array}{c}\text { Real estate, renting, computer and other } \\
\text { business activities }\end{array}$} \\
\hline Total & $\begin{array}{r}\text { Sale \& repair } \\
\text { of motor } \\
\text { vehicles \& fuel }\end{array}$ & $\begin{array}{r}\text { Other wholesale } \\
\text { trade }\end{array}$ & $\begin{array}{l}\text { Other retail } \\
\text { trade \& repair }\end{array}$ & $\begin{array}{l}\text { Hotels and } \\
\text { restaurants }\end{array}$ & $\begin{array}{r}\text { Transport, } \\
\text { storage \& } \\
\text { communication }\end{array}$ & Total & $\begin{array}{r}\text { Development, } \\
\text { buying, } \\
\text { selling, } \\
\text { renting of real } \\
\text { estate }\end{array}$ & $\begin{array}{r}\text { Renting of } \\
\text { machinery \& } \\
\text { equipment }\end{array}$ \\
\hline
\end{tabular}

Amounts outstanding (sterling \& other currencies)

\begin{tabular}{|c|c|c|c|c|c|c|c|c|c|}
\hline \multicolumn{10}{|c|}{ Loans $\&$ advances (including under repo \& sterling commercial paper) } \\
\hline & TBOR & TBOS & TBOT & TBOU & TBOV & TBOW & TBOX & TBOY & TBPA \\
\hline 2005 & 40548 & 9293 & 13312 & 17943 & 25064 & 20836 & 177152 & 137281 & 6661 \\
\hline 2006 & 42368 & 10167 & 14401 & 17800 & 25707 & 26361 & 209942 & 162332 & 6881 \\
\hline Acce & & & & & & & & & \\
\hline & TBQR & TBQS & TBQT & TBQU & TBQV & TBQW & $T B Q X$ & TBQY & TBRA \\
\hline 2005 & 151 & 7 & 120 & 25 & - & - & 721 & 714 & - \\
\hline 2006 & 160 & 4 & 99 & 58 & 1 & 1 & 812 & 800 & - \\
\hline Total & & & & & & & & & \\
\hline & TBSR & TBSS & TBST & TBSU & TBSV & TBSW & TBSX & TBSY & TBTA \\
\hline 2008 & 56778 & 9929 & 23726 & 23124 & 32260 & 32454 & 310911 & 250265 & 9931 \\
\hline 2009 & 47438 & 11260 & 15639 & 20539 & .. & 29600 & 300783 & 249284 & 7766 \\
\hline of $w$ & n sterling & & & & & & & & \\
\hline & TBUR & TBUS & TBUT & TBUU & TBUV & TBUW & TBUX & TBUY & TBVA \\
\hline 2008 & 42861 & 9292 & 12905 & 20664 & 31101 & 23257 & 290342 & 242033 & 8094 \\
\hline 2009 & 40314 & 10881 & 10313 & 19119 & 31742 & 21805 & 285896 & 242599 & 6596 \\
\hline Facili & ranted & & & & & & & & \\
\hline 2008 & 84998 & 12741 & 33528 & 38730 & 37840 & 51623 & 373289 & 292979 & 11608 \\
\hline 2009 & 73645 & 14217 & 25525 & 33903 & 38110 & 46966 & 359753 & 288737 & 9776 \\
\hline of $w$ & n sterling & & & & & & & & \\
\hline & TCCR & TCCS & ТССТ & TCCU & TCCV & TCCW & TCCX & TCCY & TCDA \\
\hline 2008 & 61914 & 11488 & 18283 & 32143 & 34908 & 32810 & 340935 & 279854 & 9136 \\
\hline 2009 & 56681 & 13066 & 15402 & 28213 & 35707 & 30663 & 332399 & 276677 & 7811 \\
\hline & $\begin{array}{l}\text { Real estate, } \\
\text { and other b }\end{array}$ & $\begin{array}{l}\text { ting, computer } \\
\text { ess activities }\end{array}$ & & & & $\begin{array}{l}\text { Recreational } \\
\text { community se }\end{array}$ & $\begin{array}{l}\text { rsonal \& } \\
\text { e activities }\end{array}$ & $\begin{array}{l}\text { Financial inte } \\
\text { insurance \& }\end{array}$ & $\begin{array}{l}\text { diation (excl. } \\
\text { sion funds) }\end{array}$ \\
\hline & $\begin{array}{r}\text { Computer \& } \\
\text { related } \\
\text { activities }\end{array}$ & $\begin{array}{r}\text { Legal, } \\
\text { accountancy, } \\
\text { consultancy \& } \\
\text { other business } \\
\text { activities }\end{array}$ & $\begin{array}{r}\text { Public } \\
\text { administration } \\
\text { \& defence }\end{array}$ & Education & $\begin{array}{r}\text { Health \& social } \\
\text { work }\end{array}$ & $\begin{array}{r}\text { Recreational, } \\
\text { cultural \& } \\
\text { sporting } \\
\text { activities }\end{array}$ & $\begin{array}{r}\text { Personal \& } \\
\text { community } \\
\text { services } \\
\text { activities }\end{array}$ & Total & $\begin{array}{r}\text { Financial } \\
\text { leasing } \\
\text { corporations }\end{array}$ \\
\hline
\end{tabular}

Amounts outstanding (sterling \& other currencies)

\begin{tabular}{|c|c|c|c|c|c|c|c|c|c|}
\hline \multicolumn{10}{|c|}{ Loans \& advances (including under repo \& sterling commercial paper) } \\
\hline & TBPB & TBPC & TBPD & TBPE & TBPF & TBPH & TBPG & TBPI & TBPJ \\
\hline 2006 & 4431 & 36299 & 17227 & 7498 & 15854 & 12255 & 5594 & 491121 & 41068 \\
\hline 2007 & .. & .. & .. & .. & .. & .. & .. & - & .. \\
\hline \multicolumn{10}{|c|}{ Acceptances } \\
\hline & TBRB & TBRC & TBRD & TBRE & TBRF & TBRH & TBRG & TBRI & TBRJ \\
\hline 2006 & - & 12 & - & - & - & - & 5 & 90 & - \\
\hline 2007 &.. &.. & .. & .. & .. &.. & .. & - & .. \\
\hline \multicolumn{10}{|l|}{ Total } \\
\hline & TBTB & TBTC & TBTD & TBTE & TBTF & ТВТН & TBTG & TBTI & TBTJ \\
\hline 2008 & 5405 & 46103 & 31792 & 10544 & 21475 & 14473 & 6801 & 832114 & 46520 \\
\hline 2009 & 4767 & 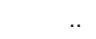 & 13270 & 11522 & 22084 & 13630 & 6686 & 700908 & 39408 \\
\hline \multicolumn{10}{|c|}{ of which in sterling } \\
\hline & TBVB & TBVC & TBVD & TBVE & TBVF & TBVH & TBVG & TBVI & TBVJ \\
\hline 2008 & 2814 & 37401 & 31282 & 10357 & 20987 & 12951 & 6010 & 489665 & 35863 \\
\hline 2009 & 3137 & 33564 & 12140 & 11371 & 21684 & 12409 & 6100 & 415759 & 28855 \\
\hline \multicolumn{10}{|c|}{ Facilities granted } \\
\hline 2008 & 7648 & 61054 & 35091 & 13882 & 24490 & 19697 & 8966 & 918210 & 50171 \\
\hline 2009 & 6896 & 54345 & 16182 & 14913 & 25324 & 17886 & 8784 & 796179 & 42778 \\
\hline \multicolumn{10}{|c|}{ of which in sterling } \\
\hline & TCDB & TCDC & TCDD & TCDE & TCDF & $\mathrm{TCDH}$ & TCDG & TCDI & TCDJ \\
\hline 2008 & 3862 & 48083 & 33733 & 13441 & 23620 & 16188 & 7686 & 515363 & 39213 \\
\hline 2009 & 4192 & 43719 & 14892 & 14663 & 24618 & 15201 & 7757 & 437238 & 31604 \\
\hline
\end{tabular}




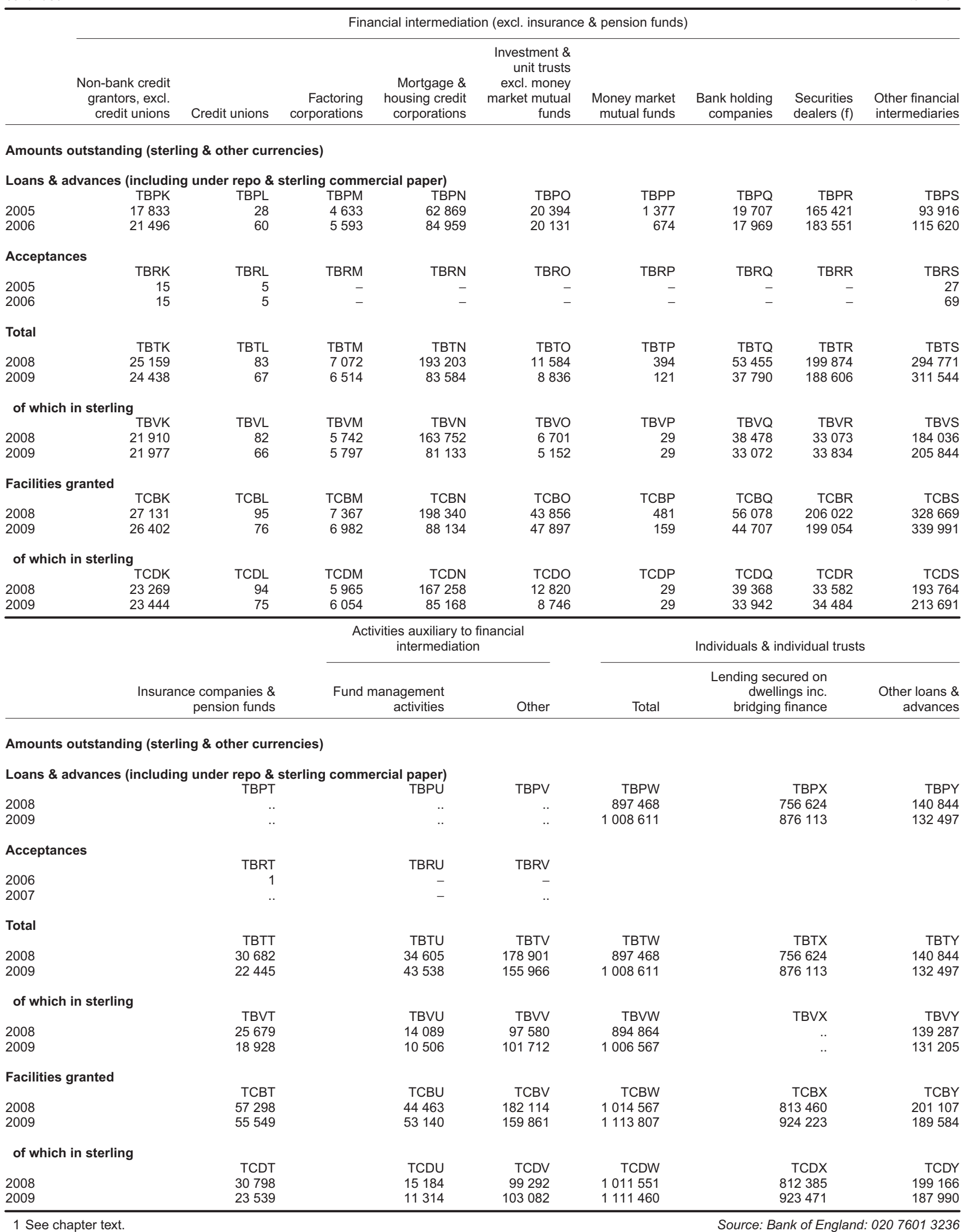




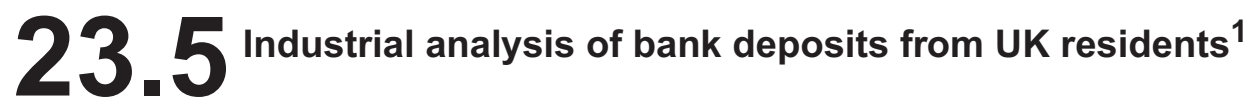

$£$ million

\begin{tabular}{|c|c|c|c|c|c|c|c|c|}
\hline & \multirow[b]{2}{*}{$\begin{array}{r}\text { Total from UK } \\
\text { residents }\end{array}$} & \multirow[b]{2}{*}{$\begin{array}{r}\text { Agriculture, } \\
\text { hunting and } \\
\text { forestry }\end{array}$} & \multirow[b]{2}{*}{ Fishing } & \multirow[b]{2}{*}{$\begin{array}{l}\text { Mining \& } \\
\text { quarrying }\end{array}$} & \multicolumn{4}{|c|}{ Manufacturing } \\
\hline & & & & & Total & $\begin{array}{r}\text { Food, beverages } \\
\& \text { tobacco }\end{array}$ & $\begin{array}{r}\text { Textiles \& } \\
\text { leather }\end{array}$ & $\begin{array}{r}\text { Pulp, paper, } \\
\text { publishing \& } \\
\text { printing }\end{array}$ \\
\hline \multicolumn{9}{|c|}{ Amounts outstanding (sterling \& other currencies) } \\
\hline \multicolumn{9}{|c|}{ Deposit liabilities (including under repos) } \\
\hline & TDAA & TDAB & TDAC & TDAD & TDAE & TDAF & TDAG & TDAH \\
\hline 2008 & 2249663 & 5265 & 175 & 10586 & 38884 & 3595 & 1121 & 3738 \\
\hline 2009 & 2314921 & 5076 & 202 & 7483 & 44808 & 3207 & 1283 & 4192 \\
\hline \multicolumn{9}{|c|}{ of which in sterling } \\
\hline & TDCA & TDCB & TDCC & TDCD & TDCE & TDCF & TDCG & $\mathrm{TDCH}$ \\
\hline 2008 & 1848495 & 5024 & 162 & 2868 & 28101 & 2370 & 850 & 3030 \\
\hline \multirow[t]{2}{*}{2009} & 1968754 & 4815 & 193 & 2185 & 31545 & 2593 & 1026 & 3505 \\
\hline & \multicolumn{5}{|c|}{ Manufacturing } & \multicolumn{2}{|c|}{ Electricity, gas and water supply } & \\
\hline
\end{tabular}

Amounts outstanding (sterling \& other currencies)

Deposit liabilities (including under repos)

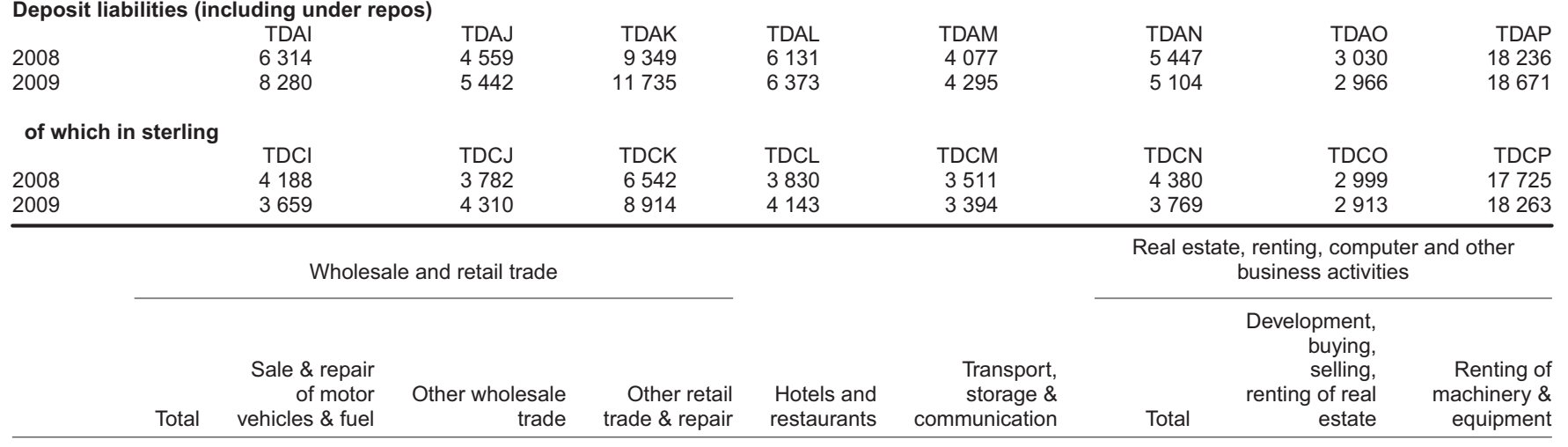

Amounts outstanding (sterling \& other currencies)

Deposit liabilities (including under repos)

\begin{tabular}{|c|c|c|c|c|c|c|c|c|c|}
\hline \multicolumn{10}{|c|}{ Deposit liabilities (in } \\
\hline & TDAQ & TDAR & TDAS & TDAT & TDAU & TDAV & TDAW & TDAX & TDAY \\
\hline 2008 & 30494 & 4063 & 13462 & 12969 & 4380 & 19347 & 121623 & 33600 & 1750 \\
\hline 2009 & 32867 & 4192 & 14282 & 14393 & 4652 & 17788 & 128596 & 35714 & 1912 \\
\hline \multicolumn{10}{|c|}{ of which in sterling } \\
\hline & TDCQ & TDCR & TDCS & TDCT & TDCU & TDCV & TDCW & TDCX & TDCY \\
\hline 2008 & 25856 & 3561 & 10288 & 12006 & 4198 & 15075 & 109549 & 32623 & 1564 \\
\hline 2009 & 28259 & 3864 & 10952 & 13443 & 4578 & 14088 & 111148 & 34944 & 1608 \\
\hline
\end{tabular}


Banking, insurance

\section{Industrial analysis of bank deposits from UK residents ${ }^{1}$ \\ continued}

$£$ million

Real estate, renting, computer

and other business activities

Legal

Computer \& consultancy \&

$\begin{array}{rr}\text { related } & \text { other business } \\ \text { activities } & \text { activities }\end{array}$

\begin{tabular}{|c|c|c|}
\hline $\begin{array}{r}\text { Public } \\
\text { administration } \\
\text { \& defence }\end{array}$ & Education & $\begin{array}{r}\text { Health \& social } \\
\text { work }\end{array}$ \\
\hline & & \\
\hline
\end{tabular}

Recreational, personal \&

community service activities

Financial intermediation (excl.

insurance \& pension funds)

Recreational, Personal \&

cultural \& community

sporting services activities activities
Financial

leasing Total corporations

Amounts outstanding (sterling \& other currencies)

Deposit liabilities (including under repos)

\begin{tabular}{|c|c|c|c|c|c|c|c|c|c|}
\hline \multicolumn{10}{|c|}{ Deposit liabilities (Includ } \\
\hline & TDAZ & TDBA & TDBB & TDBC & TDBD & TDBF & TDBE & TDBG & TDBH \\
\hline 2008 & 9547 & 76727 & 54071 & 10285 & 14525 & 16353 & 15918 & 647181 & 7356 \\
\hline 2009 & 9862 & 81107 & 57099 & 11199 & 15287 & 17562 & 16488 & 703175 & 4979 \\
\hline \multicolumn{10}{|c|}{ of which in sterling } \\
\hline & TDCZ & TDDA & TDDB & TDDC & TDDD & TDDF & TDDE & TDDG & TDDH \\
\hline 2008 & 7856 & 67506 & 50794 & 9914 & 13154 & 15234 & 15358 & 422201 & 630 \\
\hline 2009 & 8332 & 66264 & 53445 & 10731 & 14443 & 16332 & 15699 & 511014 & 3994 \\
\hline \multicolumn{10}{|c|}{ Financial intermediation (excl. insurance \& pension funds) } \\
\hline & \multicolumn{9}{|c|}{$\begin{array}{l}\text { Investment \& } \\
\text { unit trusts }\end{array}$} \\
\hline & $\begin{array}{r}\text { Non-bank credit } \\
\text { grantors, excl. } \\
\text { credit unions }\end{array}$ & Credit unions & $\begin{array}{r}\text { Factoring } \\
\text { corporations }\end{array}$ & $\begin{array}{r}\text { Mortgage \& } \\
\text { housing credit } \\
\text { corporations }\end{array}$ & $\begin{array}{r}\text { excl. money } \\
\text { market mutual } \\
\text { funds }\end{array}$ & $\begin{array}{c}\text { Money market } \\
\text { mutual funds }\end{array}$ & $\begin{array}{r}\text { Bank holding } \\
\text { companies }\end{array}$ & $\begin{array}{r}\text { Securities } \\
\text { dealers }\end{array}$ & $\begin{array}{l}\text { Other financi } \\
\text { intermediarie }\end{array}$ \\
\hline
\end{tabular}

Amounts outstanding (sterling \& other currencies)

Deposit liabilities (including under repos)

\begin{tabular}{|c|c|c|c|c|c|c|c|c|c|}
\hline \multicolumn{10}{|c|}{ labilitie } \\
\hline & TDBI & TDBJ & TDBK & TDBL & TDBM & TDBN & TDBO & TDBP & TDBQ \\
\hline 2008 & 6248 & 443 & 829 & 108501 & 37969 & 376 & 65164 & 143714 & 276582 \\
\hline 2009 & 8606 & 514 & 841 & 115982 & 34200 & 498 & 67516 & 131651 & 338387 \\
\hline \multicolumn{10}{|c|}{ of which in sterling } \\
\hline & TDDI & TDDJ & TDDK & TDDL & TDDM & TDDN & TDDO & TDDP & TDD \\
\hline 2008 & 3900 & 443 & 678 & 106490 & 23260 & 233 & 45368 & 36246 & 199278 \\
\hline 2009 & 6864 & 514 & 756 & 114225 & 25659 & 303 & 48376 & 40126 & 270198 \\
\hline
\end{tabular}

\begin{tabular}{rrrr}
\hline & Insurance companies \& pension \\
funds & Placed by fund managers & Octivies auxiliary to financial intermediation & Other
\end{tabular}

Amounts outstanding (sterling \& other currencies)

Deposit liabilities (including under repos)

\begin{tabular}{lr} 
& TDBR \\
2008 & 65978 \\
2009 & 57738 \\
& \\
of which in sterling & TDDR \\
2008 & 5563 \\
2009 & 4898 \\
\hline
\end{tabular}

\begin{tabular}{rrr} 
TDBS & TDBT & TDBU \\
111869 & 154853 & 901162 \\
88046 & 157179 & 922934 \\
& & \\
TDDS & TDDT & TDDU \\
52161 & 101373 & 896732 \\
42945 & 114721 & 918685 \\
\hline
\end{tabular}

1 See chapter text. 

23.6 $\begin{aligned} & \text { Public sector net cash requirement and other counterparts to changes in money } \\ & \text { stock during the year }\end{aligned}$

Not seasonally adjusted $£$ million

\begin{tabular}{|c|c|c|c|c|c|c|c|c|c|c|c|c|}
\hline & & 1999 & 2000 & 2001 & 2002 & 2003 & 2004 & 2005 & 2006 & 2007 & 2008 & 2009 \\
\hline $\begin{array}{l}\text { Public sector net cash requirement } \\
\text { (surplus) }\end{array}$ & ABEN & -3205 & -36864 & -2019 & 18010 & 37160 & 41915 & 41278 & 33916 & 31089 & 125369 & 20518 \\
\hline M4 lending ${ }^{1}$ & AvBS & 78029 & 111202 & 82574 & 107553 & 127820 & 156084 & 158087 & 218445 & 238491 & 270535 & 136 \\
\hline $\begin{array}{l}\text { External and foreign currency finance of the } \\
\text { public sector } \\
\text { Other external and foreign currency flows }{ }^{2} \\
\text { Net non-deposit liabilities (increase) }\end{array}$ & $\begin{array}{l}\text { VQDC } \\
\text { AVBW } \\
\text { AVBX }\end{array}$ & $\begin{array}{r}6199 \\
-44902 \\
-2943\end{array}$ & $\begin{array}{r}3616 \\
7178 \\
-31050\end{array}$ & $\begin{array}{r}3875 \\
-21631 \\
-10791\end{array}$ & $\begin{array}{r}2486 \\
-25132 \\
-25130\end{array}$ & $\begin{array}{l}-13441 \\
-27124 \\
-20377\end{array}$ & $\begin{array}{r}-2395 \\
4288 \\
-67401\end{array}$ & $\begin{array}{r}-30708 \\
33643 \\
-39903\end{array}$ & $\begin{array}{r}-33554 \\
-874 \\
-29964\end{array}$ & $\begin{array}{r}-38366 \\
-37241 \\
-4451\end{array}$ & $\begin{array}{r}-36146 \\
148653 \\
-172812\end{array}$ & $\begin{array}{r}-2117 \\
-15995 \\
-4963\end{array}$ \\
\hline Money stock (M4) & AUZI & 33329 & 67198 & 58994 & 68834 & 73271 & 100014 & 150869 & 167024 & 188522 & 258700 & 134 \\
\hline
\end{tabular}

1 Bank and building society lending, plus holdings of commercial bills by the Source: Bank of England: 02076015468

Issue Department of the Bank of England.

2 Including sterling lending to non-residents sector.

\subsection{Money stock and liquidity $^{2}$}

$£$ million

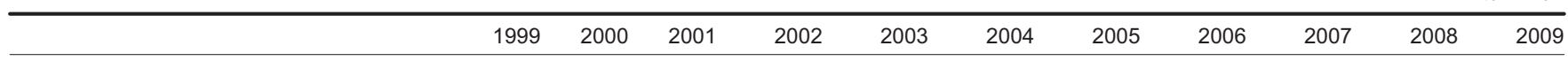

Amounts outstanding at end-year

Notes and coin in circulation with the M4 private sector ${ }^{1}$

UK private sector sterling non-interest

$\begin{array}{llllllllllll}\text { VQKT } & 26269 & 28174 & 30450 & 31889 & 34010 & 36410 & 38508 & 40539 & 43001 & 46 & 173 \quad 47952\end{array}$

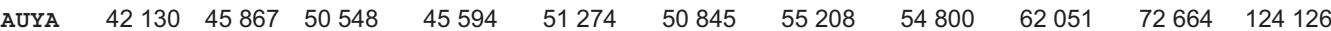

Money stock (M2) ${ }^{3} \quad$ vQxv $558334597523649980703920777347 \quad 845654922687996645107141611237531184765$

Money stock M4

AUYM 81660188487394259410087501081299117919213283211498936167484719371372048927

Changes during the year ${ }^{4}$

Notes and coin in circulation with the

M4 private sector ${ }^{1}$

UK private sector sterling non-interest

bearing sight deposits

\begin{tabular}{rrrrrrrrrrrr} 
VQLU & 2582 & 1957 & 2284 & 1493 & 2189 & 2461 & 2156 & 2053 & 2536 & 3136 & 4185 \\
AUZA & 5354 & 3533 & 4914 & -6761 & 5321 & -227 & 5699 & -409 & 9292 & 587 & 35522 \\
AUZE & 41992 & 39123 & 52813 & 53698 & 72255 & 68901 & 78428 & 72764 & 65042 & 48558 &.. \\
AUZI & 33329 & 67198 & 58994 & 68834 & 73271 & 100014 & 150869 & 167024 & 188522 & 258700 & 134380 \\
\hline
\end{tabular}

Money stock (M2) ${ }^{3}$

Money stock M4 1 The estimates of levels of coin in circulation include allowance for wastage, $\begin{array}{r}3 \mathrm{M} 2 \text { comprises the UK non-monetary financial institutions and non-public sec- } \\ \text { tor, i.e. M4 private sector's holdings of notes and coin together with its sterling }\end{array}$
hoarding, etc.

2 Non-interest bearing deposits are confined to those with institutions includdenominated retail deposits with UK monetary financial institutions.

ed in the United Kingdom banks sector (See Table 23.3).

4 As far as possible the changes exclude the effect of changes in the number of contributors to the series, and also of the introduction of new statistical returns. Changes are not seasonally adjusted. 
Banking, insurance

Selected retail banks' base rate ${ }^{1}$

Operative between dates shown

Percentage rates

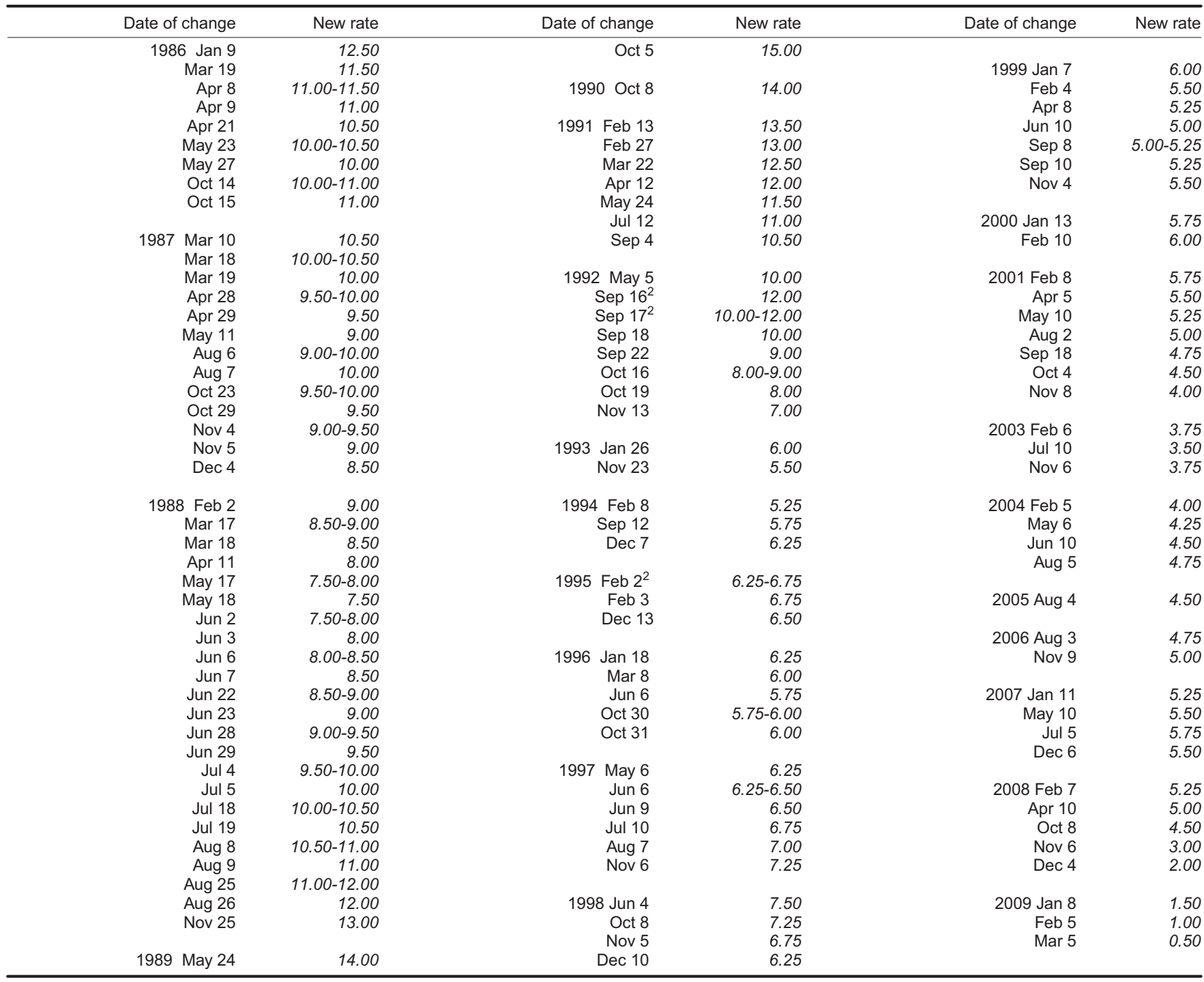

1 Data obtained from Barclays Bank, Lloyds/TSB Bank, HSBC Bank and

National Westminster Bank whose rates are used to compile this series.

Source: Bank of England: 02076014444

2 Where all the rates did not change on the same day a spread is shown. 


\begin{tabular}{|c|c|c|c|c|c|c|c|c|c|c|c|c|}
\hline & 1998 & 1999 & 2000 & 2001 & 2002 & 2003 & 2004 & 2005 & 2006 & 2007 & 2008 & 2009 \\
\hline \multicolumn{13}{|c|}{ Treasury bills: ${ }^{2}$ KDMM } \\
\hline January & 6.80 & 5.28 & 5.72 & 5.49 & 3.83 & 3.80 & 3.92 & 4.66 & 4.39 & 5.30 & 5.12 & 0.90 \\
\hline February & 6.88 & 5.04 & 5.83 & 5.46 & 3.87 & 3.50 & 4.01 & 4.69 & 4.38 & 5.34 & 5.02 & 0.72 \\
\hline March & 6.95 & 4.92 & 5.86 & 5.23 & 3.97 & 3.47 & 4.13 & 4.77 & 4.40 & 5.33 & 4.88 & 0.60 \\
\hline April & 7.00 & 4.90 & 5.92 & 5.12 & 3.97 & 3.45 & 4.20 & 4.70 & 4.42 & 5.43 & 4.83 & 0.63 \\
\hline May & 7.01 & 4.93 & 5.95 & 4.98 & 3.95 & 3.44 & 4.40 & 4.66 & 4.50 & 5.55 & 4.95 & 0.53 \\
\hline June & 7.29 & 4.76 & 5.85 & 4.99 & 3.98 & 3.47 & 4.61 & 4.62 & 4.54 & 5.67 & 5.11 & 0.50 \\
\hline July & 7.22 & 4.76 & 5.83 & 5.01 & 3.84 & 3.31 & 4.67 & 4.46 & 4.53 & 5.77 & 5.08 & 0.44 \\
\hline August & 7.19 & 4.85 & 5.81 & 4.72 & 3.77 & 3.40 & 4.71 & 4.41 & 4.75 & 5.79 & 4.95 & 0.40 \\
\hline September & 6.94 & 5.12 & 5.78 & 4.43 & 3.79 & 3.52 & 4.69 & 4.40 & 4.84 & 5.69 & 4.74 & 0.38 \\
\hline October & 6.54 & 5.23 & 5.75 & 4.16 & 3.75 & 3.65 & 4.68 & 4.40 & 4.94 & 5.61 & 3.68 & 0.43 \\
\hline November & 6.31 & 5.20 & 5.68 & 3.78 & 3.80 & 3.81 & 4.66 & 4.42 & 5.01 & 5.50 & 1.99 & 0.47 \\
\hline December & 5.72 & 5.46 & 5.62 & 3.83 & 3.84 & 3.83 & 4.68 & 4.43 & 5.08 & 5.30 & 1.29 & 0.36 \\
\hline
\end{tabular}

Eligible bill: $\mathrm{KDMY}^{3}$

$\begin{array}{lllllllll}\text { January } & 7.28 & 5.63 & 5.90 & 5.64 & 3.91 & 3.87 & 3.94 & 4.75 \\ \text { February } & 7.24 & 5.28 & 6.01 & 5.56 & 3.92 & 3.65 & 4.06 & 4.78 \\ \text { March } & 7.25 & 5.11 & 5.98 & 5.37 & 3.99 & 3.54 & 4.19 & 4.88 \\ \text { April } & 7.24 & 5.02 & 6.05 & 5.21 & 4.04 & 3.52 & 4.28 & 4.84 \\ \text { May } & 7.20 & 5.08 & 6.09 & 5.06 & 4.01 & 3.52 & 4.42 & 4.80 \\ \text { June } & 7.42 & 4.94 & 6.03 & 5.08 & 4.04 & 3.45 & 4.68 & 4.76 \\ & & & & & & & & \\ \text { July } & 7.49 & 4.89 & 5.97 & 5.07 & 3.94 & 3.39 & 4.75 & 4.57 \\ \text { August } & 7.40 & 4.94 & 5.97 & 4.82 & 3.86 & 3.42 & 4.85 & 4.51 \\ \text { September } & 7.20 & 5.16 & 5.95 & 4.57 & 3.86 & 3.59 & 4.83 & . . \\ \text { October } & 6.91 & 5.42 & 5.92 & 4.26 & 3.82 & 3.69 & 4.79 & . . \\ \text { November } & 6.52 & 5.43 & 5.88 & 3.85 & 3.84 & 3.88 & 4.78 & . . \\ \text { December } & 6.05 & 5.59 & 5.78 & 3.88 & 3.71 & 3.90 & 4.77 & . .\end{array}$

Interbank rate: AMIJ

$\begin{array}{lll}\text { January } & 7.48 & 5.8 \\ \text { February } & 7.46 & 5.43 \\ \text { March } & 7.48 & 5.3 \\ \text { April } & 7.44 & 5.23 \\ \text { May } & 7.41 & 5.2 \\ \text { June } & 7.63 & 5.1 \\ & & \\ \text { July } & 7.71 & 5.07 \\ \text { August } & 7.66 & 5.1 \\ \text { September } & 7.38 & 5.32 \\ \text { October } & 7.14 & 5.94 \\ \text { November } & 6.89 & 5.78 \\ \text { December } & 6.38 & 5.97\end{array}$

$\begin{array}{lllllllllll}5.80 & 6.06 & 5.76 & 3.98 & 3.91 & 3.99 & 4.80 & 4.54 & 5.45 & 5.61 & 2.28 \\ 5.43 & 6.15 & 5.69 & 3.98 & 3.69 & 4.10 & 4.82 & 4.52 & 5.52 & 5.61 & 2.08 \\ 5.30 & 6.15 & 5.47 & 4.06 & 3.58 & 4.23 & 4.92 & 4.53 & 5.50 & 5.86 & 1.83 \\ 5.23 & 6.21 & 5.33 & 4.11 & 3.58 & 4.33 & 4.88 & 4.57 & 5.61 & 5.90 & 1.48 \\ 5.25 & 6.23 & 5.17 & 4.08 & 3.57 & 4.46 & 4.83 & 4.65 & 5.72 & 5.79 & 1.30 \\ 5.12 & 6.14 & 5.19 & 4.11 & 3.57 & 4.73 & 4.78 & 4.69 & 5.83 & 5.90 & 1.21 \\ 5.07 & 6.11 & 5.19 & 3.99 & 3.42 & 4.79 & 4.59 & 4.68 & 5.98 & 5.80 & 1.03 \\ 5.18 & 6.14 & 4.93 & 3.92 & 3.45 & 4.89 & 4.53 & 4.90 & 6.34 & 5.76 & 0.80 \\ 5.32 & 6.12 & 4.65 & 3.93 & 3.63 & 4.87 & 4.54 & 4.98 & 6.58 & 5.87 & 0.62 \\ 5.94 & 6.08 & 4.36 & 3.90 & 3.73 & 4.83 & 4.53 & 5.09 & 6.21 & 6.18 & 0.56 \\ 5.78 & 6.00 & 3.93 & 3.91 & 3.91 & 4.82 & 4.56 & 5.18 & 6.36 & 4.40 & 0.60 \\ 5.97 & 5.89 & 3.99 & 3.95 & 3.95 & 4.81 & 4.59 & 5.25 & 6.35 & 3.21 & 0.61\end{array}$

Certificate of deposits: KOSA

\begin{tabular}{|c|c|c|c|c|c|c|c|}
\hline January & 7.44 & 5.74 & 6.02 & 5.73 & 3.96 & 3.90 & 3.98 \\
\hline February & 7.42 & 5.38 & 6.10 & 5.66 & 3.96 & 3.68 & 4.09 \\
\hline March & 7.43 & 5.26 & 6.09 & 5.44 & 4.04 & 3.57 & 4.22 \\
\hline April & 7.40 & 5.19 & 6.17 & 5.30 & 4.08 & 3.57 & 4.32 \\
\hline May & 7.37 & 5.22 & 6.19 & 5.15 & 4.06 & 3.56 & 4.45 \\
\hline June & 7.59 & 5.09 & 6.10 & 5.16 & 4.09 & 3.56 & 4.72 \\
\hline July & 7.66 & 5.03 & 6.08 & 5.17 & 3.97 & 3.41 & 4.79 \\
\hline August & 7.61 & 5.14 & 6.09 & 4.90 & 3.90 & 3.44 & 4.89 \\
\hline September & 7.34 & 5.28 & 6.08 & 4.62 & 3.91 & 3.62 & 4.87 \\
\hline October & 7.09 & 5.86 & 6.05 & 4.33 & 3.88 & 3.72 & 4.83 \\
\hline November & 6.82 & 5.72 & 5.98 & 3.91 & 3.89 & 3.90 & 4.81 \\
\hline December & 6.32 & 5.89 & 5.85 & 3.96 & 3.93 & 3.94 & 4.80 \\
\hline \multicolumn{8}{|c|}{ Local authority deposits: KDPX ${ }^{4}$} \\
\hline January & 7.43 & 5.76 & 6.03 & 5.73 & 3.85 & 3.87 & 3.91 \\
\hline February & 7.40 & 5.38 & 6.09 & 5.62 & 3.88 & 3.61 & 4.08 \\
\hline March & 7.40 & 5.27 & 6.08 & 5.39 & 4.01 & 3.55 & 4.12 \\
\hline April & 7.38 & 5.17 & 6.12 & 5.26 & 4.05 & 3.54 & 4.31 \\
\hline May & 7.34 & 5.19 & 6.14 & 5.13 & 4.06 & 3.54 & 4.45 \\
\hline June & 7.56 & 5.07 & 6.09 & 5.10 & 4.05 & 3.57 & 4.75 \\
\hline July & 7.64 & 5.01 & 6.04 & 5.12 & 3.95 & 3.39 & 4.82 \\
\hline August & 7.55 & 5.11 & 6.06 & 4.86 & 3.87 & 3.43 & 4.9 \\
\hline September & 7.35 & 5.19 & 6.05 & 4.58 & 3.88 & 3.61 & 4.90 \\
\hline October & 7.08 & 5.83 & 6.03 & 4.29 & 3.86 & 3.71 & 4.85 \\
\hline November & 6.85 & 5.64 & 5.96 & 3.82 & 3.87 & 3.90 & 4.84 \\
\hline December & 6.35 & 5.88 & 5.80 & 3.87 & 3.93 & 3.92 & 4.82 \\
\hline
\end{tabular}

1 A full definition of these series is given in Section 7 of the ONS Financial 3 This series discontinued at end of August 2005

Statistics Explanatory Handbook.

4 This series discontinued at end of December 2004

2 Average rate of discount at weekly (Friday) tender. 


\begin{tabular}{|c|c|c|c|c|c|c|c|c|c|c|c|c|}
\hline & 1998 & 1999 & 2000 & 2001 & 2002 & 2003 & 2004 & 2005 & 2006 & 2007 & 2008 & 2009 \\
\hline \multicolumn{13}{|c|}{$\begin{array}{l}\text { Sterling exchange } \\
\text { rate index }(1990=100)^{2} \text { AGBG }\end{array}$} \\
\hline January & 104.7 & 99.6 & 108.5 & 104.4 & 106.9 & 104.0 & 102.4 & 102.1 & 102.7 & .. & .. & \\
\hline February & 104.7 & 100.8 & 108.4 & 104.1 & 107.4 & 102.4 & 104.8 & 103.3 & 102.8 & .. & .. & \\
\hline March & 106.8 & 102.8 & 108.4 & 105.0 & 106.5 & 100.6 & 105.0 & 103.2 & 102.1 &.. & .. & \\
\hline April & 107.1 & 103.4 & 110.1 & 105.8 & 107.1 & 99.8 & 105.2 & 104.4 & 101.9 &.. & .. & \\
\hline May & 103.4 & 104.2 & 108.5 & 106.6 & 105.3 & 97.9 & 104.6 & 103.6 & 104.1 &.. & .. & \\
\hline June & 105.4 & 104.7 & 104.6 & 106.8 & 103.6 & 99.6 & 105.8 & 104.9 &.. &.. & .. & \\
\hline July & 105.3 & 103.5 & 105.6 & 107.2 & 105.3 & 99.4 & 105.9 & 102.1 &.. &.. & .. & \\
\hline August & 104.6 & 103.3 & 107.4 & 105.1 & 105.4 & 99.0 & 105.2 & 102.8 & .. & .. & .. & \\
\hline September & 103.3 & 104.7 & 106.2 & 106.1 & 106.5 & 99.2 & 103.3 & 103.9 &.. &.. & .. & \\
\hline October & 100.7 & 105.4 & 109.2 & 105.8 & 106.7 & 99.8 & 102.2 & 103.1 &.. &.. & .. & \\
\hline November & 100.6 & 105.7 & 107.3 & 106.1 & 105.9 & 100.4 & 101.7 & 103.2 &.. &.. & .. & \\
\hline December & 100.4 & 106.7 & 106.4 & 106.5 & 105.5 & 100.3 & 103.2 & 103.3 &.. &.. & .. & \\
\hline \multicolumn{13}{|c|}{$\begin{array}{l}\text { Effective Sterling exchange } \\
\text { rate index (Jan } 2005=100) \text { BK67 }\end{array}$} \\
\hline January & 100.4 & 96.3 & 102.9 & 98.5 & 100.6 & 99.9 & 100.2 & 100.0 & 99.1 & 105.4 & 96.5 & 76.7 \\
\hline February & 100.1 & 97.1 & 102.5 & 98.1 & 100.8 & 98.5 & 102.5 & 101.0 & 98.9 & 104.9 & 96.0 & 78 \\
\hline March & 101.8 & 98.8 & 102.3 & 98.8 & 100.1 & 96.8 & 102.2 & 101.1 & 98.4 & 103.4 & 94.5 & 76.6 \\
\hline April & 102.1 & 98.8 & 103.7 & 99.4 & 100.9 & 96.1 & 102.2 & 102.0 & 98.4 & 104.1 & 92.7 & 78.6 \\
\hline May & 98.9 & 99.6 & 101.7 & 99.8 & 99.5 & 94.9 & 101.8 & 101.0 & 101.2 & 103.8 & 92.7 & 80.2 \\
\hline June & 100.9 & 99.6 & 98.7 & 99.6 & 98.2 & 96.6 & 103.1 & 101.8 & 100.9 & 104.4 & 92.8 & 83.7 \\
\hline July & 100.8 & 98.4 & 99.4 & 100.2 & 100.4 & 96.1 & 103.2 & 98.8 & 100.9 & 105.1 & 92.9 & 83.2 \\
\hline August & 100.3 & 98.7 & 100.6 & 98.8 & 100.3 & 95.5 & 102.4 & 99.7 & 102.9 & 104.4 & 91.4 & 83.4 \\
\hline September & 99.6 & 99.8 & 99.1 & 99.9 & 101.4 & 95.8 & 100.7 & 100.7 & 102.9 & 103.2 & 89.6 & 80.9 \\
\hline October & 97.4 & 100.7 & 101.7 & 99.7 & 101.7 & 96.9 & 99.8 & 99.7 & 103.1 & 102.7 & 89.1 & 79.1 \\
\hline November & 97.2 & 100.6 & 100.1 & 99.8 & 101.1 & 97.5 & 99.7 & 99.4 & 103.4 & 101.7 & 83.2 & 80.7 \\
\hline December & 97.2 & 101.3 & 99.8 & 100.4 & 100.8 & 97.9 & 101.3 & 99.5 & 104.4 & 99.8 & 77.9 & 80.1 \\
\hline \multicolumn{13}{|c|}{ Sterling/US Dollar AUSS } \\
\hline January & 1.6 & 1.7 & 1.6 & 1.5 & 1.4 & 1.6 & 1.8 & 1.9 & 1.8 & 2.0 & 2.0 & 1. \\
\hline February & 1.6 & 1.6 & 1.6 & 1.5 & 1.4 & 1.6 & 1.9 & 1.9 & 1.7 & 2.0 & 2.0 & 1. \\
\hline March & 1.7 & 1.6 & 1.6 & 1.4 & 1.4 & 1.6 & 1.8 & 1.9 & 1.7 & 1.9 & 2.0 & 1.4 \\
\hline April & 1.7 & 1.6 & 1.6 & 1.4 & 1.4 & 1.6 & 1.8 & 1.9 & 1.8 & 2.0 & 2.0 & 1.5 \\
\hline May & 1.6 & 1.6 & 1.5 & 1.4 & 1.5 & 1.6 & 1.8 & 1.9 & 1.9 & 2.0 & 2.0 & 1.5 \\
\hline June & 1.7 & 1.6 & 1.5 & 1.4 & 1.5 & 1.7 & 1.8 & 1.8 & 1.8 & 2.0 & 2.0 & 1.6 \\
\hline July & 1.6 & 1.6 & 1.5 & 1.4 & 1.6 & 1.6 & 1.8 & 1.8 & 1.8 & 2.0 & 2.0 & 1. \\
\hline August & 1.6 & 1.6 & 1.5 & 1.4 & 1.5 & 1.6 & 1.8 & 1.8 & 1.9 & 2.0 & 1.9 & 1 \\
\hline September & 1.7 & 1.6 & 1.4 & 1.5 & 1.6 & 1.6 & 1.8 & 1.8 & 1.9 & 2.0 & 1.8 & 1 \\
\hline October & 1.7 & 1.7 & 1.5 & 1.5 & 1.6 & 1.7 & 1.8 & 1.8 & 1.9 & 2.0 & 1.7 & 1.6 \\
\hline November & 1.7 & 1.6 & 1.4 & 1.4 & 1.6 & 1.7 & 1.9 & 1.7 & 1.9 & 2.1 & 1.5 & 1.7 \\
\hline December & 1.7 & 1.6 & 1.5 & 1.4 & 1.6 & 1.8 & 1.9 & 1.7 & 2.0 & 2.0 & 1.5 & 1.6 \\
\hline
\end{tabular}

Sterling/Euro THAP

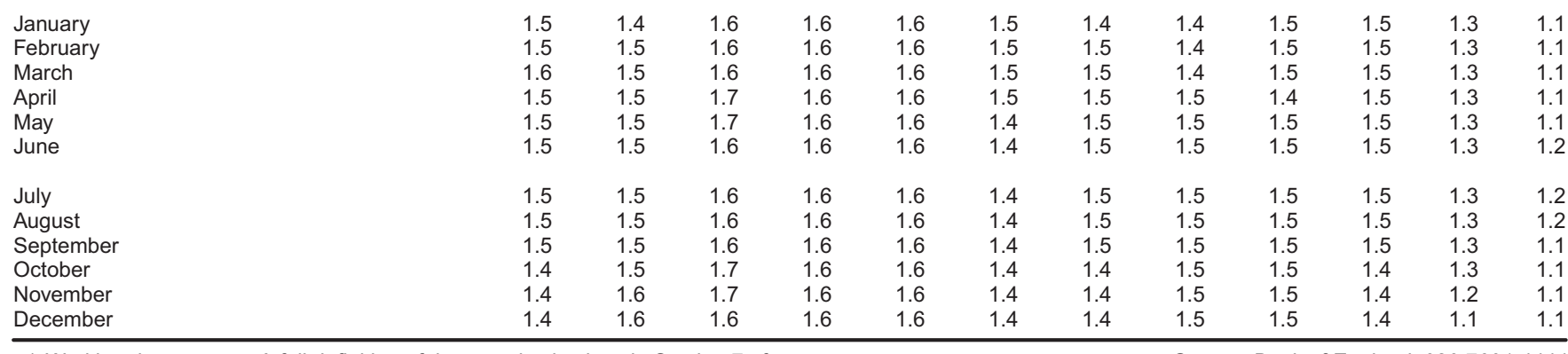

1 Working day average. A full definition of these series is given in Section 7 of

Source: Bank of England: 02076014444 2 Series discontinued from 31 May 2006. 


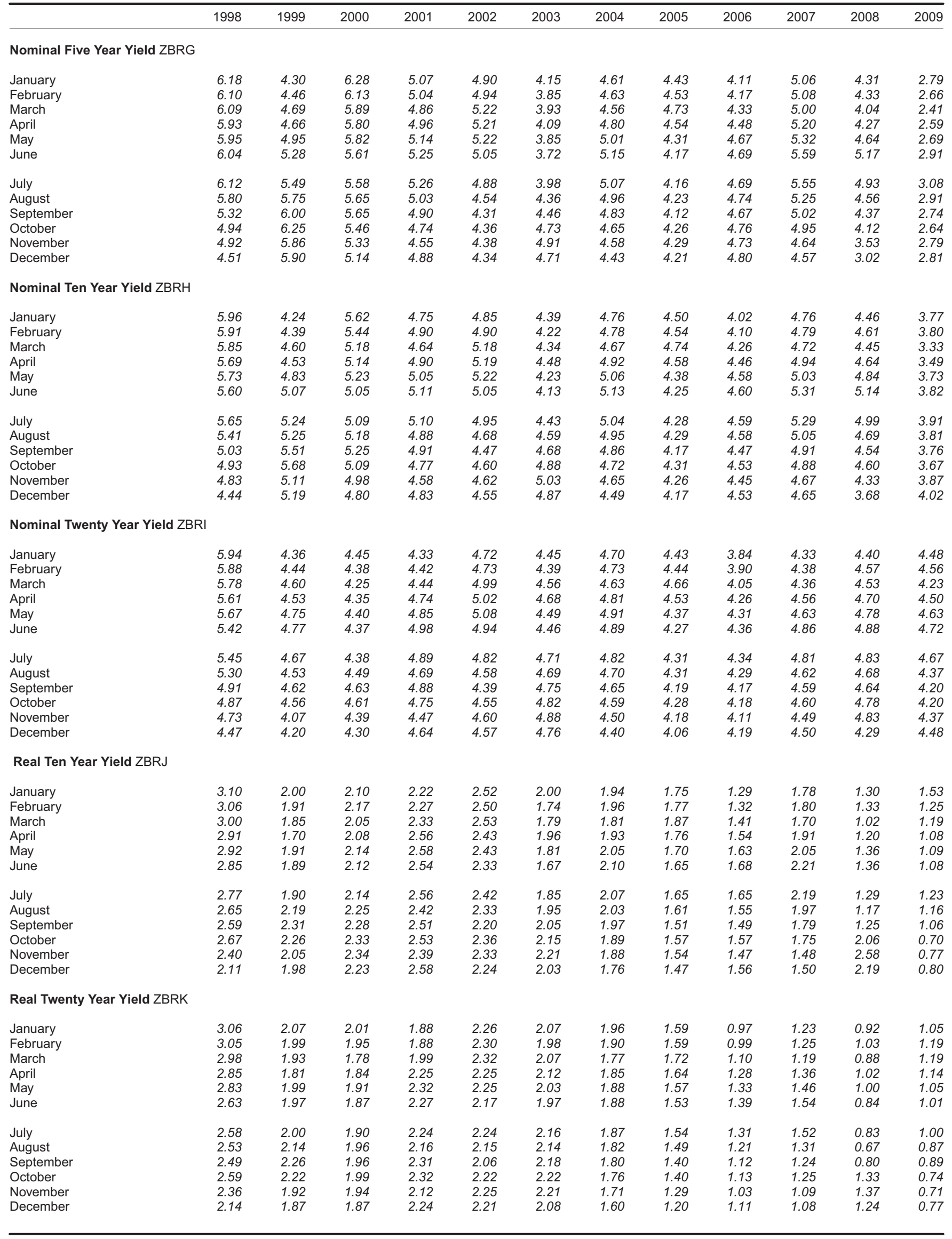




\subsection{Average rates on representative British Government Stocks ${ }^{1}$}

Percentage rates

199

$19992000 \quad 2001$

2002

2003

2004

2005

2006

2007

$2008 \quad 2009$

5 Year Conventional Rate ${ }^{2}$ KORP

January

February

March

April

May

June

July

August

September

October

November

December
6.33

6.24

6.26

6.11

6.14

4.93

6.14

5.84

4.88
4.86

4.45

04

\subsection{5 \\ 6.36}

4.41

4.65
4.66

4.93

$5.49 \quad 5.75$

5.80

6.24
5.89

5.89
5.91
6.23
6.01

5.95

5.75
5.81

5.81

5.66

5.50
5.27

\section{$5.17 \quad 4.94$}

5.13

4.94
4.97

5.15

5.32

5.34

4.94

4.78

4.59
4.94
4.96

5.23
5.26
5.48

5.10

4.92
4.57

4.57
4.25

4.38

4.40
4.34

\subsection{5}

3.88
3.93

4.08

3.83

3.68

3.72

4.30
4.42

4.42

4.70
4.88

4.68

4.59

4.46

4.44

4.66

5.08

4.98

4.88

4.76

4.57

4.52
4.42

\subsection{3}

4.61
4.77

4.58

4.36

4.24

4.11
4.22

4.18

4.23

4.31

4.27

10 year Conventional Rate KORQ

January

February

March

April

May

June

July

August

September

October

November

December

20 Year Conve

6.07
6.02
5.97
5.81
5.85
5.77

$\begin{array}{ll}4.16 & 5.75 \\ 4.32 & 5.56\end{array}$

$4.54 \quad 5.29$

$4.48 \quad 5.25$

$4.77 \quad 5.35$
5.02

$5.02 \quad 5.15$

$5.20 \quad 5.18$

5.67
5.56

5.56
5.10

5.10
4.93

4.87

5.24

5.52

5.70

5.16

5.27

5.32
5.15

5.06

$$
\begin{aligned}
& 4.86 \\
& 4.88 \\
& 4.75 \\
& 4.95 \\
& 5.13 \\
& 5.09 \\
& \\
& 5.16 \\
& 4.92 \\
& 4.92 \\
& 4.76 \\
& 4.58 \\
& 4.88
\end{aligned}
$$

4.86
4.88
4.75
4.95
5.13
5.09

5.16
4.92
4.92
4.76
4.58
4.88

4.84
4.91
5.15
5.23
5.51
5.06

4.94
4.66
4.46
4.57
4.59

4.84
4.91
5.15
5.23
5.51
5.06

4.94
4.66
4.46
4.57
4.59
4.52

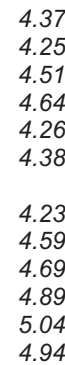

4.37
4.25
4.51
4.64
4.26
4.38

4.23
4.59
4.69
4.89
5.04
4.94

4.78
4.75
4.65
4.91
5.07
5.19

5.10
4.99
4.89
4.73
4.66
4.50

4.78
4.75
4.65
4.91
5.07
5.19

5.10
4.99
4.89
4.73
4.66
4.50

4.51
4.60
4.79
4.60
4.38
4.23
4.20
4.25
4.16
4.31
4.33
4.27

4.51
4.60
4.79
4.60
4.38
4.23

4.20
4.25
4.16
4.31
4.33
4.27

4.27

4.31

4.41
4.44

4.44

4.66

4.62

4.84

5.00

4.39
January

March

April

May

June

July

August

September

October

November

December

6.04

5.98

5.90
5.73

5.79

4.36

4.47

4.64

4.58

4.83

4.92

5.63

5.43
5.02

5.02
4.92

4.79

4.49

4.88
4.82

4.97

4.97

4.46
4.56

10 Year Index-Linked Rate KORS

January

February

March

April

May

June

July

August

October

November

December

3.01

2.94
2.89

2.89

2.80

2.83
2.81

2.00

1.94

1.90

1.96
1.93

1.93

2.20

2.32

2.26

2.03
1.99

20 Year Index-Linked rate KORT

January

February

March

April

May

June

July

August

September

October

November

December

1 Working day average.

2 Discontinued from 6 December 2006.

$\begin{array}{llllllllll}4.91 & 4.52 & 4.81 & 4.46 & 4.73 & 4.55 & 4.05 & 4.77 & 4.54 & 3.72 \\ 4.80 & 4.58 & 4.83 & 4.37 & 4.80 & 4.58 & 4.13 & 4.80 & 4.68 & 3.85 \\ 4.64 & 4.56 & 5.12 & 4.51 & 4.69 & 4.79 & 4.28 & 4.73 & 4.54 & 3.48 \\ 4.71 & 4.84 & 5.14 & 4.64 & 4.91 & 4.63 & 4.38 & 4.95 & 4.77 & 3.48 \\ 4.77 & 4.98 & 5.45 & 4.44 & 5.03 & 4.43 & 4.59 & 5.08 & 4.95 & 3.63 \\ 4.68 & 5.10 & 5.03 & 4.38 & 5.07 & 4.30 & 4.60 & 5.32 & 5.22 & 3.79 \\ 4.70 & 5.05 & 4.92 & 4.59 & 4.99 & 4.33 & 4.60 & 5.28 & 5.09 & 3.77 \\ 4.79 & 4.83 & 4.65 & 4.67 & 4.88 & 4.34 & 4.58 & 5.04 & 4.62 & 3.76 \\ 4.90 & 4.94 & 4.46 & 4.74 & 4.83 & 4.24 & 4.49 & 4.89 & 4.67 & 3.79 \\ 4.84 & 4.80 & 4.59 & 4.85 & 4.73 & 4.37 & 4.53 & 4.92 & 4.65 & 3.68 \\ 4.64 & 4.55 & 4.65 & 4.93 & 4.64 & 4.31 & 4.48 & 4.73 & 4.33 & 3.83 \\ 4.51 & 4.75 & 4.61 & 4.80 & 4.53 & 4.22 & 4.41 & 4.71 & 3.70 & 3.95\end{array}$

$\begin{array}{llllllllll}2.11 & 2.21 & 2.61 & 2.07 & 1.88 & 1.73 & 1.48 & 2.24 & 1.60 & 1.77 \\ 2.16 & 2.30 & 2.53 & 1.81 & 1.90 & 1.81 & 1.54 & 2.27 & 1.29 & 1.18 \\ 2.06 & 2.34 & 2.55 & 1.88 & 1.76 & 1.99 & 1.65 & 2.18 & 0.76 & 0.83 \\ 2.08 & 2.55 & 2.45 & 1.90 & 1.94 & 1.83 & 1.65 & 2.61 & 1.12 & 0.79 \\ 2.15 & 2.61 & 2.58 & 1.74 & 2.10 & 1.71 & 1.96 & 2.59 & 1.48 & 0.47 \\ 2.13 & 2.56 & 2.35 & 1.59 & 2.17 & 1.67 & 1.94 & 2.73 & 1.77 & 0.53 \\ & & & & & & & & & \\ 2.14 & 2.57 & 2.46 & 1.67 & 2.12 & 1.66 & 1.92 & 2.68 & 1.61 & 0.13 \\ 2.25 & 2.45 & 2.37 & 1.89 & 2.04 & 1.63 & 1.80 & 2.37 & 1.64 & 0.01 \\ 2.29 & 2.56 & 2.24 & 1.99 & 1.95 & 1.49 & 1.76 & 2.01 & 1.69 & - \\ 2.33 & 2.55 & 2.42 & 2.08 & 1.83 & 1.89 & 1.89 & 1.99 & 2.38 & - \\ 2.32 & 2.42 & 2.39 & 2.16 & 1.85 & 1.64 & 1.87 & 1.70 & 3.44 & -0.72 \\ 2.20 & 2.65 & 2.30 & 1.97 & 1.74 & 1.27 & 1.96 & 1.80 & 3.59 & -1.26\end{array}$
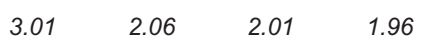

2.35

$2.10 \quad 1.95$

1.99

2.07
2.10

2.00
1.93

1.94

1.80

1.91
1.99

2.01

$2.05 \quad 1.99$

$\begin{array}{ll}2.05 & 1.99 \\ 2.09 & 1.93 \\ 2.13 & 1.89\end{array}$

$2.17 \quad 1.84$

2.16

2.04

1.80
1.69
1.68

1.72

1.89
1.77

1.67
1.63

1.63

1.63

1.58
1.48

1.48
1.51

1.45

1.38

$\begin{array}{llll}1.17 & 1.63 & 1.25 & 1.57 \\ 1.22 & 1.65 & 1.30 & 1.33 \\ 1.34 & 1.57 & 1.04 & 1.27 \\ 1.38 & 1.77 & 1.24 & 1.18 \\ 1.56 & 1.91 & 1.38 & 1.20 \\ 1.61 & 2.07 & 1.34 & 1.78 \\ & & & \\ 1.55 & 2.07 & 1.27 & 1.26 \\ 1.45 & 1.83 & 1.14 & 1.23 \\ 1.37 & 1.67 & 1.26 & 1.10 \\ 1.44 & 1.65 & 1.99 & 0.74 \\ 1.34 & 1.41 & 2.47 & 0.82 \\ 1.43 & 1.41 & 2.14 & 0.87\end{array}$




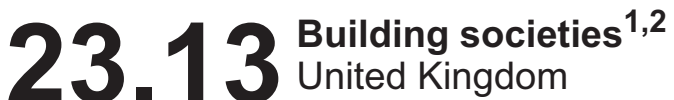

\begin{tabular}{|c|c|c|c|c|c|c|c|c|c|c|c|}
\hline & & $1999^{3}$ & $2000^{4}$ & 2001 & 2002 & 2003 & 2004 & 2005 & 2006 & 2007 & $2008^{5}$ \\
\hline \multicolumn{12}{|l|}{ Number and balance sheets } \\
\hline Societies on register (numbers) & KRNA & 72 & 68 & 65 & 65 & 63 & 63 & 63 & 60 & 59 & 59 \\
\hline Share investors (thousands) & KRNB & 21774 & 22237 & 20311 & 20724 & 20901 & 20734 & 22090 & 22396 & 23038 & 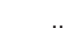 \\
\hline Depositors (thousands) & KRNC & 642 & 660 & 501 & 440 & 452 & 446 & 370 & 391 & 387 & .. \\
\hline Borrowers (thousands) & KRND & 2868 & 2925 & 2579 & 2520 & 2520 & 2570 & 2617 & 2626 & 2642 & \\
\hline
\end{tabular}

Assets and liabilities ( $£$ million)

Liabilities:
Shares
Deposits and wholesale
Taxation and other
General reserves
Other Capital
Assets:
Mortgages
Investments and cash
Other
Total

\begin{tabular}{|c|c|c|c|c|c|c|c|c|c|c|}
\hline KRNE & 109137.7 & 119298.5 & 119815.2 & 132372.9 & 142477.1 & 153844.0 & 171935.0 & 188943.0 & 206782.5 & 224424.0 \\
\hline KRNF & 34746.6 & 44262.4 & 37358.9 & 37933.0 & 49552.6 & 64025.2 & 70845.1 & 75443.0 & 92097.6 & 100633.2 \\
\hline KRNG & 1665.4 & 1664.0 & 1244.9 & 1088.4 & 1179.0 & 1394.9 & 2619.4 & 6838.5 & 10703.3 & 9583.3 \\
\hline KRNH & 8301.5 & 8987.1 & 8511.2 & 9043.4 & 9489.8 & 10123.9 & 10677.4 & 10845.3 & 12080.3 & 11620.7 \\
\hline KRNI & 1529.2 & 1861.0 & 1416.1 & 1709.2 & 2534.7 & 3599.1 & 4566.5 & 5510.9 & 6073.8 & \\
\hline RNK & 123183.4 & 137072.3 & 130229.6 & 140839.7 & 159938.2 & 184191.0 & 2076 & 2311 & 265 & 269313.4 \\
\hline KHVZ & 29917.8 & 36574.2 & 35925.9 & 38952.7 & 43067.9 & 46234.1 & 49240.3 & 52349.4 & 57843.5 & 72477.7 \\
\hline KRNN & 2279.2 & 2426.6 & 2190.7 & 2354.4 & 2226.9 & 2562.0 & 3781.7 & 4032.3 & 4555.9 & 8100.4 \\
\hline & 0.4 & 3.0 & 583 & 21 & 5 & 32 & 606 & 87 & 32 & 349891.5 \\
\hline
\end{tabular}

Current transactions $(£$ million

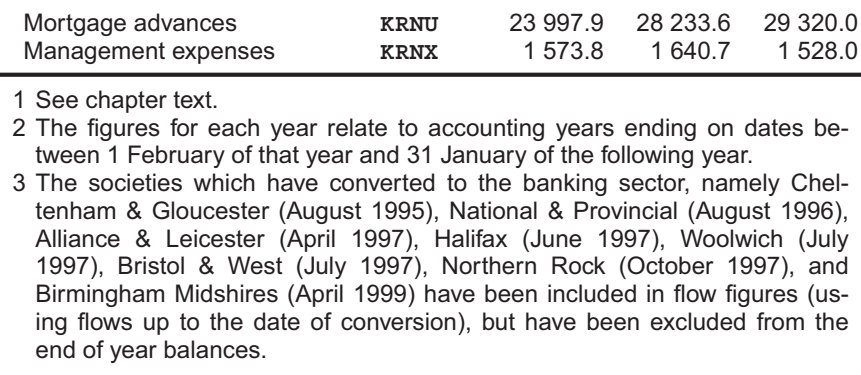

$\begin{array}{lllllll}33077.0 & 43392.4 & 51089.0 & 50059.4 & 52327.5 & 61678.8 & 47215.6\end{array}$ 4 Bradford \& Bingley, which converted to the banking sector in December 2000, is included within flow figures and the end of year balances.

52008 Reporting requirement changes - data is now sourced from QFS1 rather than AFS1. Mortgages Advances taken from MLAR (Mortgage Lenders and Administrators return)

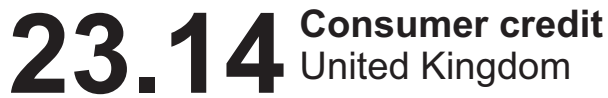

\begin{tabular}{|c|c|c|c|c|c|c|c|c|c|c|c|}
\hline & & 2000 & 2001 & 2002 & 2003 & 2004 & 2005 & 2006 & 2007 & 2008 & 2009 \\
\hline Total amount outstanding & VZRD & 135168 & 150802 & 169209 & 180649 & 198856 & 211038 & 212835 & 221687 & 233164 & 226827 \\
\hline Total net lending & VZQC & 15965 & 19673 & 23443 & 22401 & 25337 & 19666 & 13054 & 13471 & 10866 & -713 \\
\hline \multicolumn{12}{|l|}{ of which } \\
\hline Credit cards & vzQs & 6686 & 6229 & 7579 & 8710 & 9998 & 6166 & 1951 & 2251 & 4092 & 2326 \\
\hline Other & VZQT & 9280 & 13445 & 15867 & 13692 & 15340 & 13499 & 11102 & 11221 & 6774 & -3039 \\
\hline Banks & AIKN & 13217 & 16055 & 17452 & 15269 & 19370 & 11317 & 9346 & 6075 & 3529 & -1622 \\
\hline Building societies' class 3 loans & ALPY & 112 & 63 & 180 & 177 & 172 & 238 & 217 & 260 & 132 & 7 \\
\hline Other consumer credit lenders & BM59 & 2640 & 3554 & 5811 & 6954 & 5796 & 8112 & 3489 & 7135 & 7205 & 901 \\
\hline Total gross lending & VZQG & 160744 & 177452 & 196451 & 207255 & 221318 & 217467 & 207460 & 204632 & 193149 & 170513 \\
\hline
\end{tabular}

As from Dec 2006 the Bank of England has ceased to update the separate data on consumer credit provided by other specialist lenders, retailers and insurance companies previously contained in these tables. These categories have been merged into 'other consumer credit lenders'. 
Banking, insurance

\section{End-year assets and liabilities of investment trust companies, unit trusts ${ }^{1}$ and 23. 15 property unit trusts ${ }^{2}$}

\begin{tabular}{|c|c|c|c|c|c|c|c|c|c|c|c|}
\hline & & 1999 & 2000 & 2001 & 2002 & 2003 & 2004 & 2005 & 2006 & 2007 & 2008 \\
\hline \multicolumn{12}{|l|}{ Investment trust companies } \\
\hline Cash and UK bank deposits & AHAG & 1227 & 2202 & 2513 & 1821 & 1346 & 1756 & 1483 & 1785 & 1731 & 2017 \\
\hline Other short-term assets & CBPN & 1097 & 1082 & 656 & 805 & 1189 & 1344 & 1549 & 353 & 1572 & 1380 \\
\hline Short-term liabilities & -CBPS & -2253 & -2861 & -3008 & -2626 & -2462 & -2234 & -2111 & -2293 & -2109 & -2046 \\
\hline $\begin{array}{l}\text { Medium and long-term liabilities and } \\
\text { capital: }\end{array}$ & $-\mathrm{CBPO}$ & -57616 & -60412 & -54630 & -38054 & -48076 & -48627 & -55076 & -51426 & -57508 & -41270 \\
\hline Issued share and loan capital & $-\mathrm{CBPQ}$ & -8565 & -8934 & -8796 & -8711 & -9873 & -8210 & -7155 & -5492 & -5659 & -3834 \\
\hline Foreign currency borrowing & - CBPR & -880 & -994 & -933 & -780 & -682 & -607 & -839 & -1043 & -1118 & -1407 \\
\hline Other borrowing & $-\mathrm{CBQA}$ & -1716 & -2503 & -3251 & -2246 & -2181 & -1728 & -1420 & -1447 & -1377 & -1245 \\
\hline Reserves and provisions, etc & $-\mathrm{AHBC}$ & -46455 & -47981 & -41650 & -26317 & -35340 & -38082 & -38082 & -43444 & -49354 & -34784 \\
\hline Investments: & CBPM & 56491 & 59948 & 54822 & 37748 & 48035 & 47212 & 53265 & 50052 & 55608 & 39079 \\
\hline $\begin{array}{l}\text { British government securities } \\
\text { UK company securities: }\end{array}$ & AHBF & 1217 & 821 & 645 & 471 & 303 & 466 & 769 & 533 & 715 & 628 \\
\hline $\begin{array}{l}\text { Loan capital and preference } \\
\text { shares }\end{array}$ & CBGZ & 1425 & 1654 & 1516 & 946 & 1079 & 1270 & 673 & 1071 & 1259 & 813 \\
\hline $\begin{array}{l}\text { Ordinary and deferred shares } \\
\text { Overseas company securities: }\end{array}$ & CBGY & 28010 & 33456 & 30338 & 19475 & 23292 & 23941 & 25037 & 22870 & 23034 & 14366 \\
\hline $\begin{array}{l}\text { Loan capital and preference } \\
\text { shares }\end{array}$ & $\mathrm{CBHA}$ & 979 & 963 & 1143 & 458 & 603 & 682 & 937 & 741 & 1038 & 623 \\
\hline Ordinary and deferred shares & AHCC & 23330 & 21355 & 19476 & 14453 & 20294 & 18967 & 23065 & 21659 & 25795 & 18385 \\
\hline Other investments & CBPT & 1530 & 1699 & 1704 & 1945 & 2464 & 1886 & 2784 & 3178 & 3767 & 4264 \\
\hline \multicolumn{12}{|l|}{ Unit trusts } \\
\hline Short-term assets and liabilities: & CBPU & 5894 & 8340 & 7979 & 8041 & 10256 & 10229 & 13944 & 18023 & 22247 & 26526 \\
\hline Cash and UK bank deposits & AGYE & 4797 & 6969 & 5748 & 5321 & 5243 & 6302 & 7740 & 12336 & 16443 & 16257 \\
\hline Other short-term assets & CBPW & 1545 & 2319 & 2763 & 3072 & 5990 & 4390 & 7420 & 6990 & 9036 & 15699 \\
\hline Short-term liabilities & $-\mathrm{CBPX}$ & -448 & -948 & -532 & -352 & -977 & -463 & -1216 & -1303 & -3232 & -5430 \\
\hline Foreign currency borrowing & -AGYK & - & - & - & - & - & - & - & - & - & - \\
\hline Investments: & CBPZ & 213553 & 222844 & 204899 & 210002 & 245516 & 269064 & 351645 & 420153 & 457729 & 388282 \\
\hline $\begin{array}{l}\text { British government securities } \\
\text { UK company securities: }\end{array}$ & СВHT & 3627 & 4693 & 4690 & 7077 & 9125 & 9768 & 25181 & 31603 & 32120 & 33466 \\
\hline $\begin{array}{l}\text { Loan capital and preference } \\
\text { shares }\end{array}$ & $\mathrm{CBHU}$ & 13322 & 14654 & 16318 & 21152 & 23972 & 22467 & 29293 & 29876 & 30626 & 30174 \\
\hline $\begin{array}{l}\text { Loan capital and preference } \\
\text { shares }\end{array}$ & CBHV & 3032 & 3212 & 4113 & 5916 & 9840 & 13142 & 16057 & 25617 & 30029 & 30442 \\
\hline Ordinary and deferred shares & RLIC & 70256 & 79601 & 71329 & 63152 & 75074 & 81034 & 105443 & 127409 & 142211 & 113667 \\
\hline Other assets & CBQE & 3820 & 3876 & 4800 & 9997 & 11098 & 12801 & 18522 & 20011 & 27734 & 36983 \\
\hline \multicolumn{12}{|l|}{ Property unit trusts } \\
\hline Short-term assets and liabilities (net) & AGVC & 205 & 285 & 247 & 242 & 459 & 466 & 686 & 1258 & 785 & 592 \\
\hline Property & CBQG & 2722 & 3488 & 2078 & 4026 & 5125 & 5909 & 9623 & 12781 & 12480 & 8518 \\
\hline Other assets & AGVL & 436 & 380 & 151 & 677 & 373 & 1366 & 1864 & 2713 & 1648 & 1040 \\
\hline Long-term borrowing & $-A G V M$ & -75 & -391 & -90 & -75 & -76 & -63 & -250 & -90 & -158 & -177 \\
\hline
\end{tabular}

Note: Assets are shown as positive: liabilities as negative.

Source: Office for National Statistics: 01633456635

1 Including open ended investment companies (OEICs).

2 Investments are at market value.

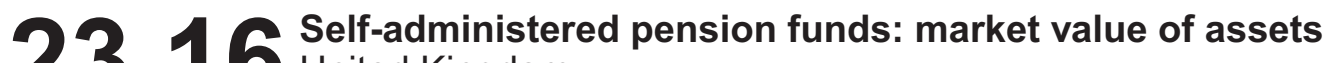

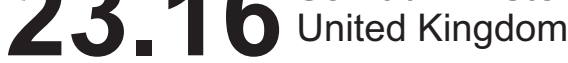 End year}

$£$ million

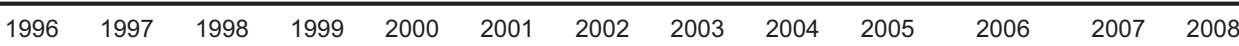

\begin{tabular}{|c|c|c|c|c|c|c|c|c|c|c|c|c|c|c|}
\hline \multicolumn{15}{|l|}{ Total pension funds ${ }^{1}$} \\
\hline Total net assets & AHVA & . & r & 促 & -26 & (3) & 1158 & 610441 & 692694 & 761066 & 91495 & 010794 & 1023979 & 864606 \\
\hline Short-term assets & RYIQ & 31521 & 35368 & 39005 & 32703 & 36638 & 31337 & 30700 & 46091 & 57476 & 73649 & 98691 & 99681 & 96546 \\
\hline British government securities & AHVK & 57783 & 80533 & 91084 & 98882 & 92458 & 83754 & 84461 & 88803 & 87579 & 94325 & 104910 & 113617 & 98577 \\
\hline ong-term debt & AHVO & 89 & 156 & 83 & 33 & 177 & 125 & 42 & 8 & 4 & 4 & 2 & 5 & \\
\hline Ordinary shares & AHVP & 01 & 339687 & 334 & 357230 & 2993 & 260 & 186 & 186 & & 19 & $20 \varepsilon$ & 152048 & 110571 \\
\hline Other & AHVQ & & & & & & & & & & & & & \\
\hline \multicolumn{15}{|l|}{ Overseas company securities } \\
\hline 证 & AHVS & 909 & 3851 & 3842 & 5099 & 12736 & 781 & 386 & 12475 & 996 & 502 & 536 & 45470 & 48222 \\
\hline UK lo & RLDQ & 83 & 0 & 22 & 14 & 7 & 3 & - & 35 & 44 & 6 & 6 & 12 & \\
\hline UK & AHWÃ & 21637 & 24176 & 24355 & 31107 & 32945 & 17 & 31658 & 30619 & 552 & 13 & 94 & 30304 & 22816 \\
\hline A & AHVU & 21767 & 21979 & 305 & 33731 & 34 & 38 & 36530 & 62029 & 82 & & 38 & 134176 & 102685 \\
\hline Pro & AHVW & & 32 & 3 & 98 & 4 & 80 & 5869 & 6761 & 444 & 87 & 889 & 16757 & 11504 \\
\hline & RKPL & 30628 & 32978 & 47136 & 82273 & 90 & 90139 & 82490 & 107229 & 152170 & 197 & 224907 & 251028 & 231234 \\
\hline Total liabilities & GQFX & 5347 & 8118 & 7436 & 8719 & 11041 & 10819 & 10005 & 26944 & 39626 & 55320 & 77108 & 68692 & 63117 \\
\hline
\end{tabular}

1 These figures cover funded schemes only and therefore exclude the main superannuation arrangements in the central government sector. 


$1999 \quad 2000 \quad 2001 \quad 2002 \quad 2003 \quad 2004$

2005

2006

2007

2008

Long-term insurance companies

Assets

Total current assets (gross)

Agents' and reinsurance balances (net)

Other debtors ${ }^{1}$

British government securities

UK local authority securities etc

UK company securities ${ }^{2}$

Overseas company securities

Overseas government securities

Loans and mortgages

UK land, property and ground rent

Overseas land, property and ground rent

Other investments

Total

Net value of direct investment in:

Non-insurance subsidiaries and associate

companies in the United Kingdom

UK associate and subsidiary insurance

companies and insurance holding companies RYEU

Overseas subsidiaries and associates $\quad$ RYEV

Total assets

RKBI

$3045 \quad 6133 \quad 4486 \quad 4577 \quad 4191$

$2245 \quad 3586 \quad 4206 \quad 4569 \quad 5054$

$3638 \quad 4002 \quad 5581 \quad 5463 \quad 6330$

96092296848195288289358894580810036401159307128242713792321190674

$8390 \quad 13016$

9186

11484

3473

2528

6114

7578

7890

\footnotetext{
Liabilities

Borrowing:

Borrowing from UK banks

Other UK borrowing

Borrowing from overseas

Long-term business:

Funds

Claims admitted but not paid

Provision for taxation net of amounts receivable:

UK authorities

Overseas authorities

Provision for recommended dividends

Other creditors and liabilities

Excess of assets over above liabilities:

Excess of value of assets over liabilities in respect of long-term funds

Minority interests in UK subsidiary companies Shareholders' capital and reserves in respect of general business

Other reserves including profit and loss account balances
}

RGDF

RGDE

RGDD

RKDC

RKBM

RYPI

RYPJ

RYPK

RYPL

RKBR

RKBR
RKTI

RKBS

RKBT

RKBI

$$
\begin{aligned}
& 6064 \\
& 3070
\end{aligned}
$$$$
\begin{aligned}
& 8272 \\
& 2823
\end{aligned}
$$

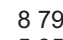

$8790 \quad 4958 \quad 4164$

$\begin{array}{rr}4958 & 4164 \\ 7406 & 10923\end{array}$

5358

8385
793

$\begin{array}{lll}9 & 036 \\ 1 & 151\end{array}$

2862
9542

9542
1965

3795

6705

5083

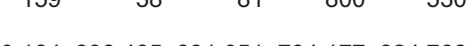

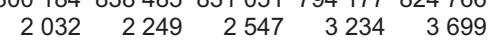

8730711037658112522112051831069993

$\begin{array}{rrrrr}3579 & 3481 & 3513 & 3848 & 3426\end{array}$

$\begin{array}{lllll}6344 & 5381 & 3951 & 2803 & 4055\end{array}$

$\begin{array}{llrrr}314 & 67 & 45 & -20 & 4881\end{array}$

201

-13
93

93
16738

8225

825
-2

-2
22
907

7908

7908
199

13

7457

457
5
27
527

54

$\begin{array}{llllllllll}116951 & 79173 & 63337 & 36517 & 62546 & 65641 & 59132 & 71017 & 75066 & 16131\end{array}$

$\begin{array}{rrrrrrrrrr}25 & - & - & - & 1 & 267 & - & - & 192 & 16131\end{array}$

$\begin{array}{llllllllll}6139 & 10287 & 17044 & 18629 & 15698 & 20719 & 18717 & 27315 & 35855 & 28443\end{array}$

$2396 \quad 2492 \quad 2130 \quad 1791 \quad 3527$

$-354 \quad 7305$ 
1998

1999

2000

2001

2002

2003

2004

2005

2006

Other than long-term insurance companies

Assets

Total current assets (gross)

Agents' and reinsurance balances (net)

Other debtors

British government securities

UK local authority securities etc

UK company securities ${ }^{2}$

Overseas company securities

Overseas government securities

Loans and mortgages

UK land, property and ground rent

Overseas land, property and ground rent

Other investments

Total

\begin{tabular}{|c|c|c|c|c|c|c|c|c|c|c|c|}
\hline RYME & 8524 & 10468 & 8772 & 12264 & 17671 & 20036 & 29258 & 26561 & 24942 & 25810 & 27563 \\
\hline AHMX & 10528 & 12177 & 8362 & .. & 9492 & 9890 &.. & .. & .. & 9932 & 10706 \\
\hline RKPS & 6277 & 7059 & 7179 & & 14437 & 13255 & & .. & .. & 20351 & 18297 \\
\hline AHMJ & 16409 & 15938 & 14561 & 15064 & 18390 & 19645 & 19662 & 19818 & 19296 & 16026 & 18441 \\
\hline AHMN & 14 & 10 & 8 & 6 & 10 & 10 & 49 & 44 & - & 3 & 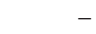 \\
\hline RKPT & 18440 & 18800 & 18585 & .. & 15362 & 15153 & .. & .. & .. & 23812 & 24204 \\
\hline RKPU & 8676 & 6284 & 8190 & & 7394 & 7124 & & . & 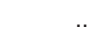 & 14773 & 20258 \\
\hline AHMS & 10459 & 7980 & 6849 & 7134 & 7156 & 5720 & 6662 & 7341 & 8035 & 4869 & 8505 \\
\hline RKPV & 1335 & 1070 & 1429 & & 1063 & 1400 & & .. & .. & 3684 & 3971 \\
\hline AHMW & 1146 & 1085 & 1069 & 860 & 805 & 859 & 893 & 1470 & 1569 & 1870 & 950 \\
\hline RYNK & 107 & 83 & 45 & 4 & 1 & 4 & 5 & 13 & 137 & 116 & \\
\hline RKPW & 2366 & 2638 & 2294 & .. & 2182 & 1408 & .. & .. & .. & 1882 & 2562 \\
\hline RKAL & 84281 & 84027 & 77343 & 78789 & 93965 & 94504 & 115356 & 116200 & 128590 & 123128 & 136544 \\
\hline RYNR & 5553 & 7074 & 7038 & 10456 & 11706 & 13408 & 19028 & 20530 & 20111 & 21954 & 212 \\
\hline RYNS & 6424 & 5617 & 5400 & 8837 & 7190 & 2918 & 2280 & 6071 & 4745 & 6936 & 7669 \\
\hline RYNT & 14239 & 17775 & 15993 & 14260 & 9014 & 5718 & 5507 & 6446 & 9657 & 9445 & 10815 \\
\hline RKBY & 110497 & 114493 & 105774 & 112342 & 121875 & 116548 & 142171 & 149247 & 163103 & 161463 & 176287 \\
\hline
\end{tabular}

Net value of direct investment in:

Non-insurance subsidiaries and associate companies in the United Kingdom

UK associate and subsidiary insurance

companies and insurance holding companies

Overseas subsidiaries and associates

RKBY

Total assets

\begin{tabular}{|c|c|c|c|c|c|c|c|c|c|c|c|}
\hline RYMB & 1825 & 1392 & 783 & 481 & 1384 & 2046 & 4519 & 893 & 3148 & 675 & 343 \\
\hline RYMC & 1551 & 3186 & 4239 & 10621 & 10472 & 9342 & 10261 & 11080 & 10445 & 10885 & 13179 \\
\hline RYMD & 1600 & 3045 & 1867 & 1964 & 2916 & 2918 & 2476 & 2817 & 5459 & 7037 & 6692 \\
\hline RKCT & 60775 & 59455 & 60236 & 60995 & 62776 & 63463 & 67241 & 71710 & 77221 & 71146 & 76980 \\
\hline RKTF & - & - & - & - & - & - & - & - & - & - & \\
\hline RKTK & - & - & - & - & - & - & - & - & - & - & \\
\hline RYPO & 1197 & 939 & 874 & 594 & 941 & 834 & 1094 & 1796 & 2376 & 2259 & 809 \\
\hline RYPP & 11 & 11 & 11 & 7 & 5 & 84 & 24 & 5 & 10 & 5 & 1 \\
\hline RYPQ & 1318 & 1817 & 2682 & 1957 & 958 & 1082 & 1311 & 5 & 270 & 222 & 48 \\
\hline RYPR & 3793 & 4981 & 6293 & 6410 & 8025 & 9567 & 10817 & 10718 & 16226 & 22069 & 21149 \\
\hline RKCG & - & - & - & - & - & - & - & - & - & - & \\
\hline $\mathrm{RKCH}$ & 68 & 29 & 33 & 276 & 4 & 6 & 6 & - & - & 599 & 1902 \\
\hline RKCI & 34397 & 35372 & 24699 & 26190 & 31982 & 25153 & 39695 & 43264 & 42186 & 38145 & 47225 \\
\hline RKCJ & 4215 & 4265 & 4056 & 2847 & 2411 & 2053 & 4727 & 6959 & 5762 & 8421 & 7959 \\
\hline KBY & 110497 & 114493 & 105774 & 112342 & 121875 & 116548 & 142171 & 149247 & 163103 & 161463 & 1762 \\
\hline
\end{tabular}

Source: Office for National Statistics: 01633456626

1 Including outstanding interest, dividends and rents (net).

Total liabilities

2 Including authorised unit trust units.

\section{Liabilities}

Other UK borrowing

General business technical reserves

ong-term business:

Provision for taxation net of amounts receivable: UK authorities

Overseas authorities

Provision for recommended dividends

Other creditors and liabilities

Exess of assets over above liabilities:

respect of long-term funds (in respect of general business

Other reserves including profit and loss account balances 


\begin{tabular}{|c|c|c|c|c|c|c|c|c|c|c|c|c|}
\hline & & \multicolumn{11}{|r|}{ Numbers } \\
\hline & & 1999 & 2000 & 2001 & 2002 & 2003 & 2004 & 2005 & 2006 & 2007 & 2008 & $2009^{7}$ \\
\hline \multicolumn{13}{|l|}{ England and Wales } \\
\hline \multirow{2}{*}{$\begin{array}{l}\text { Bankruptcies }{ }^{1} \\
\text { Individual voluntary } \\
\text { arrangements }{ }^{2,3}\end{array}$} & AIHW & 21611 & 21550 & 23477 & 24292 & 28021 & 35898 & 47291 & 62956 & 64480 & 67428 & 74670 \\
\hline & AIHI & 7195 & 7978 & 6298 & 6295 & 7583 & 10752 & 20293 & 44332 & 42165 & 39116 & 47641 \\
\hline Total & AIHK & 28806 & 29528 & 29775 & 30587 & 35604 & 46651 & 67584 & 107288 & 106645 & 106544 & 134142 \\
\hline \multicolumn{13}{|l|}{ Scotland } \\
\hline Sequestrations ${ }^{4}$ & KRHA & 3195 & 2965 & 3048 & 3215 & 3328 & 3297 & 4965 & 5430 & 6219 & 12370 & 14356 \\
\hline Protected Trust Deeds & GJ2I & 2144 & 2801 & 3779 & 5174 & 5452 & 6024 & 6881 & 8208 & 7595 & 7542 & 9126 \\
\hline Total & GJ2J & 5339 & 5766 & 6827 & 8389 & 8780 & 9321 & 11846 & 13638 & 13814 & 19912 & 23482 \\
\hline \multirow{2}{*}{$\begin{array}{l}\text { Bankruptcies }{ }^{5} \\
\text { Individual voluntary } \\
\text { arrangements }\end{array}$} & KRHB & 401 & 349 & 292 & 334 & 517 & 666 & 821 & 1035 & 898 & 1079 & 1237 \\
\hline & KJRK & 172 & 267 & 176 & 207 & 318 & 449 & 633 & 774 & 440 & 559 & 722 \\
\hline Total & KRHD & 573 & 616 & 468 & 541 & 835 & 1115 & 1454 & 1809 & 1338 & 1638 & 1959 \\
\hline
\end{tabular}

1 Comprises receiving and administration orders under the Bankruptcy Act 5 Comprises bankruptcy adjudication orders, arrangement protection orders and 1914 and bankruptcy orders under the Insolvency Act 1986. Orders later orders for the administration of estates of deceased insolvents. Orders later set consolidated or rescinded are included in these figures.

2 Introduced under the Insolvency Act 1986. aside or dismissed are included in these figures.

3 For statistical purposes deeds of arrangement are now included with indi- 7 Provisional

vidual voluntary arrangements.

4 Sequestrations awarded but not brought into operation are included in these figures.

Source: Insolvency Service: 0207637 6504/6443

\section{Company insolvencies \\ 23. 19 United Kingdom}

Numbers

\begin{tabular}{|c|c|c|c|c|c|c|c|c|c|c|c|c|}
\hline & & 1999 & 2000 & 2001 & 2002 & 2003 & 2004 & 2005 & 2006 & 2007 & 2008 & $2009^{2}$ \\
\hline \multicolumn{13}{|l|}{ England and Wales } \\
\hline $\begin{array}{l}\text { Compulsory liquidations } \\
\text { Creditors' voluntary }\end{array}$ & AIHR & 5209 & 4925 & 4675 & 6231 & 5234 & 4584 & 5233 & 5418 & 5165 & 5494 & 5643 \\
\hline liquidations & AIHS & 9071 & 9392 & 10297 & 10075 & 8950 & 7608 & 7660 & 7719 & 7342 & 10041 & 13434 \\
\hline Total & AIHQ & 14280 & 14317 & 14972 & 16306 & 14184 & 12192 & 12893 & 13137 & 12507 & 15535 & 19077 \\
\hline \multicolumn{13}{|l|}{ Scotland } \\
\hline $\begin{array}{l}\text { Compulsory liquidations } \\
\text { Creditors' voluntary }\end{array}$ & KRGA & 364 & 344 & 378 & 556 & 436 & 431 & 420 & 416 & 439 & 437 & 432 \\
\hline liquidations & KRGB & 208 & 239 & 224 & 232 & 195 & 190 & 149 & 133 & 100 & 87 & 152 \\
\hline Total & KRGC & 572 & 583 & 602 & 788 & 631 & 621 & 569 & 549 & 539 & 524 & 584 \\
\hline \multicolumn{13}{|l|}{ Northern Ireland ${ }^{1}$} \\
\hline $\begin{array}{l}\text { Compulsory liquidations } \\
\text { Creditors' voluntary }\end{array}$ & KRGD & .. & .. & .. & 49 & 95 & 76 & 85 & 78 & 122 & 158 & 164 \\
\hline liquidations & KRGE &.. &.. &.. & 53 & 47 & 45 & 53 & 50 & 42 & 51 & 83 \\
\hline Total & KRGF &.. & .. & .. & 102 & 142 & 121 & 138 & 128 & 164 & 209 & 247 \\
\hline
\end{tabular}

1 Prior to 2002, the quality of the statistics on company liquidations in

Northern Ireland are not robust enough and have been removed from this

Source: Insolvency Service: 0207637 6504/6443

table.

2 Provisional. 


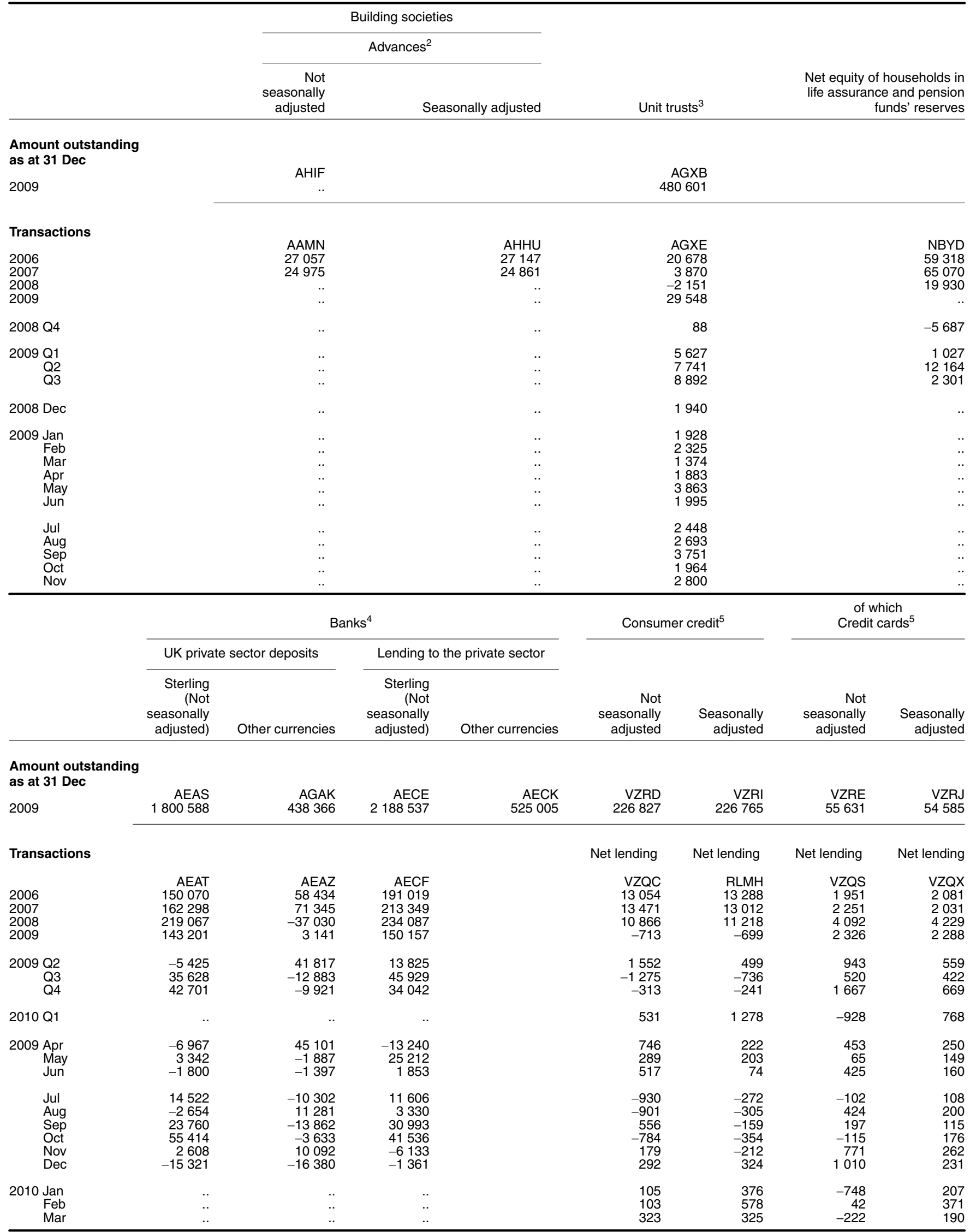

1 For further details see Financial Statistics, Tables 1.2E, 3.2B, 4.2A, 4.3A, 5 Data have been revised back to February 2003 due to the inclusion of some 4.3B, 5.2D, 6.2A, 10.5D.

2 Total administered by the Department for National Savings.

3 Including open ended investment companies (OEICs). additional other specialist lenders and the removal of some non-resident based securitisation vehicles.

4 Monthly figures relate to calendar months.

Sources: Office for National Statistics; Department for National Savings; Building Societies Commission; Association of Unit Trusts and Investment Funds Bank of England; 


\subsection{Selected interest rates, exchange rates and security prices}

\begin{tabular}{|c|c|c|c|c|c|c|}
\hline & $\begin{array}{r}\text { Selected retail } \\
\text { banks' base rate }\end{array}$ & $\begin{array}{r}\text { Average discount rate } \\
\text { for } 91 \text { day Treasury } \\
\text { bills }\end{array}$ & $\begin{array}{r}\text { Inter bank } 3 \text { months } \\
\text { bid rate }\end{array}$ & $\begin{array}{r}\text { Inter bank } 3 \text { months } \\
\text { offer rate }\end{array}$ & $\begin{array}{r}\text { British government } \\
\text { securities } 20 \text { years } \\
\text { yield }^{1}\end{array}$ & Exchange rate US spot \\
\hline 2006 Feb & 4.50 & 4.39 & 4.51 & 4.53 & 3.96 & 1.7511 \\
\hline Mar & 4.50 & 4.41 & 4.54 & 4.56 & 4.15 & 1.7345 \\
\hline Apr & 4.50 & 4.45 & 4.60 & 4.63 & 4.32 & 1.8179 \\
\hline May & 4.50 & 4.51 & 4.66 & 4.68 & 4.43 & 1.8712 \\
\hline Jun & 4.50 & 4.54 & 4.71 & 4.73 & 4.46 & 1.8494 \\
\hline Jul & 4.50 & 4.58 & 4.73 & 4.74 & 4.45 & 1.8671 \\
\hline Aug & 4.75 & 4.77 & 4.94 & 4.95 & 4.42 & 1.9018 \\
\hline Sep & 4.75 & 4.87 & 5.02 & 5.05 & 4.29 & 1.8682 \\
\hline Oct & 4.75 & 4.98 & 5.14 & 5.16 & 4.35 & 1.9073 \\
\hline Nov & 5.00 & 5.04 & 5.20 & 5.22 & 4.27 & 1.9670 \\
\hline Dec & 5.00 & 5.11 & 5.26 & 5.29 & 4.33 & 1.9570 \\
\hline 2007 Jan & 5.25 & 5.37 & 5.54 & 5.55 & 4.51 & 1.9574 \\
\hline Feb & 5.25 & 5.31 & 5.48 & 5.50 & 4.59 & 1.9600 \\
\hline Apr & 5.25 & 5.47 & 5.66 & 5.70 & 4.72 & 1.9997 \\
\hline May & 5.50 & 5.59 & 5.76 & 5.78 & .. & 1.9782 \\
\hline Jun & 5.50 & 5.77 & 5.93 & 5.98 & .. & 2.0064 \\
\hline Jul & 5.75 & 5.75 & 6.00 & 6.02 & .. & 2.0322 \\
\hline Aug & 5.75 & 5.77 & 6.55 & 6.65 & 4.80 & 2.0171 \\
\hline Sep & 5.75 & 5.61 & 6.18 & 6.28 & 4.74 & 2.0374 \\
\hline Oct & 5.75 & 5.57 & 6.17 & 6.25 & 4.74 & 2.0774 \\
\hline Nov & 5.75 & 5.44 & 6.53 & 6.58 & 4.59 & 2.0561 \\
\hline Dec & 5.50 & 5.24 & 5.95 & 5.95 & 4.59 & 1.9909 \\
\hline 2008 Jan & 5.50 & 5.01 & 5.50 & 5.58 & 4.46 & 1.9882 \\
\hline Feb & 5.25 & 4.98 & 5.68 & 5.72 & 4.62 & 1.9892 \\
\hline Mar & 5.25 & 4.77 & 5.95 & 6.02 & 4.54 & 1.9875 \\
\hline Apr & 5.00 & 4.90 & 5.76 & 5.84 & 4.73 & 1.9803 \\
\hline May & 5.00 & 5.04 & 5.80 & 5.87 & 4.85 & 1.9762 \\
\hline Jun & 5.00 & 5.10 & 5.88 & 5.94 & 5.03 & 1.9901 \\
\hline Jul & 5.00 & 5.09 & 5.75 & 5.79 & 4.94 & 1.9810 \\
\hline Aug & 5.00 & 4.94 & 5.70 & 5.75 & 4.74 & 1.8237 \\
\hline Nov & 3.00 & 1.68 & 3.85 & 4.10 & 4.69 & 1.5345 \\
\hline Dec & 2.00 & 1.24 & 2.75 & 2.90 & 4.15 & 1.4376 \\
\hline 2009 Jan & 1.50 & 0.91 & 2.00 & 2.25 & 4.28 & 1.4416 \\
\hline Feb & .. & 0.66 & 1.95 & 2.15 & 4.34 & 1.4255 \\
\hline Mar & .. & 0.62 & 1.60 & 1.70 & 4.01 & 1.4331 \\
\hline Apr & .. & 0.57 & 1.30 & 1.45 & 4.24 & 1.4820 \\
\hline May & .. & 0.52 & 1.15 & 1.30 & 4.38 & 1.6125 \\
\hline Jun & .. & 0.49 & 1.15 & 1.20 & 4.47 & 1.6468 \\
\hline Jul & .. & 0.43 & 0.90 & 0.90 & 4.46 & 1.6579 \\
\hline Aug & .. & 0.37 & 0.70 & 0.70 & 4.22 & 1.6311 \\
\hline Sep & .. & 0.39 & 0.55 & 0.60 & 4.07 & 1.5993 \\
\hline Oct & .. & 0.46 & 0.50 & 0.70 & 4.05 & 1.6478 \\
\hline Nov & .. & 0.46 & 0.50 & 0.70 & 4.22 & 1.6411 \\
\hline Dec & .. & 0.49 & 0.55 & 0.70 & 4.33 & 1.6148 \\
\hline 2010 Jan & .. & 0.49 & 0.50 & 0.70 & 4.42 & 1.6020 \\
\hline Feb & .. & 0.49 & 0.50 & 0.70 & 4.52 & 1.5224 \\
\hline Mar & .. & 0.51 & 0.50 & 0.70 & 4.57 & 1.5167 \\
\hline
\end{tabular}




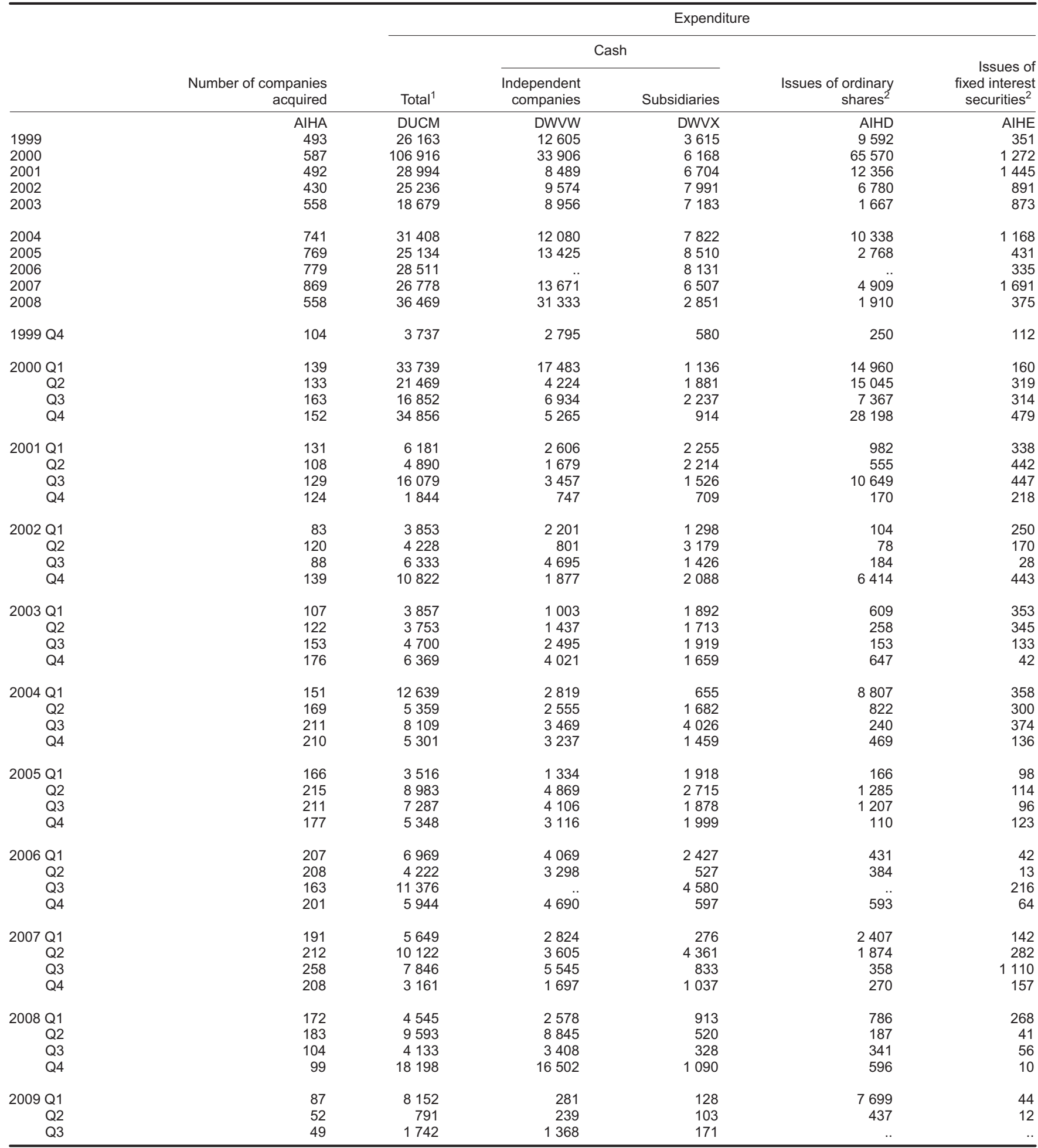

Missing data for any series have been suppressed to avoid the disclosure of information relating to individual enterprises.

2 Issued to the vendor as payment. 